\title{
molecular
} microbiology

\section{Phospho-regulation and nucleocytoplasmic trafficking of CrzA in response to calcium and alkaline-pH stress in Aspergillus nidulans.}

\begin{tabular}{|r|l|}
\hline Journal: & Molecular Microbiology \\
\hline Manuscript ID: & MMI-2013-13093.R1 \\
\hline Manuscript Type: & Research Article \\
\hline Date Submitted by the Author: & n/a \\
\hline Complete List of Authors: & $\begin{array}{l}\text { Hernández-Ortiz, Patricia; Centro de Investigaciones Biológicas CSIC, } \\
\text { Cellular and Molecular Biology } \\
\text { Espeso, Eduardo; Centro de Investigaciones Biológicas CSIC, Cellular and } \\
\text { Molecular Biology }\end{array}$ \\
\hline Key Words: & $\begin{array}{l}\text { abiotic stress response, calcineurin, GSK3beta, Casein kinase I, Aspergillus } \\
\text { nidulans }\end{array}$ \\
\hline &
\end{tabular}

SCHOLARONE ${ }^{m}$

Manuscripts 
1 Phospho-regulation and nucleocytoplasmic trafficking of $\mathrm{CrzA}$ in response to 2 calcium and alkaline-pH stress in Aspergillus nidulans

3

4

5 Hernández-Ortiz, Patricia and Espeso, Eduardo A.*.

6

7 Address:

8 Department of Cellular and Molecular Biology.

9 Centro Investigaciones Biológicas, CSIC.

10 Ramiro de Maeztu, 9. Madrid, 28040. Spain.

11 email: eespeso@cib.csic.es

* Corresponding Author

14

15 Running title: Calcium and alkaline pH Signaling of CrzA.

18 Key words. Abiotic Stress. Nucleocytoplasmic transport. Calcineurin. Phosphorylation. Casein 19 kinase. Glycogen synthase kinase 3beta. 
1 Abstract

3 Tolerance to abiotic stresses by microorganisms require of appropriate signaling and 4 regulatory pathways. Calcineurin phosphatases mediate calcium-dependent signaling 5 pathways which are widely distributed among phylogeny. In Saccharomyces cerevisiae, calcineurin mediates the post-translational modification of downstream effectors, most of them transcription factors, being the best characterized the calcineurin-regulated zincfinger factor 1, Crz1p. Here we study the signaling process of CrzA, a filamentous fungal

9 Crz orthologue, in response to calcium and ambient-pH alkalinization. In Aspergillus nidulans resting cells CrzA locates in the cytoplasm being excluded from nuclei. CrzA is a phospho-protein and upon calcium, manganese or alkaline-pH stresses, accumulates in nuclei in a calcineurin-dependent manner. Functional analysis of CrzA defined the presence of a nuclear-export and two nuclear-localization signals as well as a PSINVE 14 sequence that constitutes the major calcineurin-docking-domain. First 450 amino acids of 15 CrzA contain these functional motifs and in this region is where phosphorylated residues 16 locate. Different phosphorylation steps are identified in CrzA and activities of casein 17 kinase 1 homologue, CkiA, and of glycogen synthase kinase-3 $\beta$, identified for the first time 18 here as GskA, are involved. The phospho-signaling process and nucleocytoplasmic trafficking of CrzA shows similarities to those described in yeast for Crz1p homologues and of NFATs in mammals. 
Introduction.

2

3 Calcium $\left(\mathrm{Ca}^{2+}\right)$ signaling plays a major role in the regulation of diverse physiological responses 4 in either prokaryotic or eukaryotic organisms, although the regulatory machinery is being 5 mainly studied in eukaryotic cells. $\mathrm{Ca}^{2+}$ is an ubiquitous secondary messenger participating in 6 many vital biological processes such as cell cycle, differentiation or ion homeostasis but intracellular $\mathrm{Ca}^{2+}$ levels must be carefully regulated because excessive concentrations can lead to cell death (reviewed in (Clapham, 2007)). An essential $\mathrm{Ca}^{2+}$-signaling mediator is calmodulin $(\mathrm{CaM}) . \mathrm{Ca}^{2+}$ binds to $\mathrm{CaM}$ triggering structural rearrangements which allow the interaction of $\mathrm{CaM}$ with its effectors. A key target of $\mathrm{CaM}$ is the serine-threonine protein phosphatase type $\mathrm{B}$ (PP2B) calcineurin (Chin and Means, 2000). Calcineurin, Cn, is a heterodimer composed of a catalytic A subunit (CnA) and a regulatory B subunit (CnB) (Guerini, 1997).

Activation of phosphatase calcineurin requires binding of $\mathrm{Ca}^{2+}$ to the regulatory $\mathrm{CnB}$ subunit and of the $\mathrm{Ca}^{2+}-\mathrm{CaM}$ complex to the CaM-binding domain present in $\mathrm{CnA}$ (Guerini, 1997). Efficient dephosphorylation by calcineurin implies targeting and interaction with an specific sequence in its substrates, named the calcineurin-docking domain (CDD). Two classes of docking sites for calcineurin are identified, the consensus sequence PxIxIT and the LxVP motif (reviewed in (Li et al., 2011)).

In mammalian cells, among calcineurin substrates is the nuclear factor of activated $\mathrm{T}$ cells (NFAT). Dephosphorylation by calcineurin promotes NFAT translocation to the nucleus, where these factors become transcriptionaly active (Crabtree and Olson, 2002). Phosphorylation maintains NFATs inactive in resting cells and this post-translational process is accomplished by several constitutive kinases including casein kinase 1 (CK1), glycogen synthase kinase-3 $\beta$ (GSK3 $\beta$ ) and the dual specificity tyrosine phosphorylation regulated kinase (DYRK) (Beals et al., 1997;Gwack et al., 2006;Zhu et al., 1998). The final cellular localization of NFATs is the result of an unbalanced phosphorylation/dephosphorylation process of nuclear localization (NLS) and nuclear export (NES) sequences present in these factors (Okamura et al., 2000).

In fungi, the model yeast Saccharomyces cerevisiae has been extensively used to understand calcium signaling. The best characterized target of calcineurin is the transcription factor (TF) Crzlp, a protein unrelated to NFATs but sharing common regulatory mechanisms. In response to stress, the $\mathrm{Ca}^{2+} /$ calmodulin/calcineurin pathway is activated leading to the dephosphorylation of Crzlp and therefore its translocation to the nucleus to modulate gene expression. As in NFATs signaling, the nucleocytoplasmic trafficking of Crzlp depends on the phosphorylation state of its NLS and NES (reviewed in (Cyert, 2003)).

A CDD of the PxIxIT class mediates the interaction of Crzlp with calcineurin (Boustany and Cyert, 2002;Roy et al., 2007). Although calcineurin is the only phosphatase involved in the signaling of Crzlp, phosphorylation is carried out by different kinases. Casein kinase I, Hrr25p, 
1 and cAMP-dependent protein kinase $\mathrm{A}$ activities are responsible for preventing the activity and 2 nuclear accumulation of Crzlp (Kafadar et al., 2003;Kafadar and Cyert, 2004).

3 Calcineurin and Crzlp are required for cell growth under physiological stresses such as high 4 salinity, alkaline $\mathrm{pH}$ and cell wall damage (Cyert, 2003). Many studies have focused on 5 calcineurin function and regulation as well as the molecular mechanism that govern the activity 6 of the phosphatase in fungal models. However, a complete catalog of calcineurin downstream 7 effectors remains to be determined (Fox and Heitman, 2002;Stie and Fox, 2008). The 8 understanding of the physiological role of calcineurin signaling components is being addressed 9 by several investigations in yeasts and filamentous fungi ((Rispail et al., 2009) and references therein). Particularly the work done in Aspergillus species showed that, CrzA, the orthologue of Crz1p in $A$. nidulans, mediates tolerance to $\mathrm{Ca}^{2+}, \mathrm{Mn}^{2+}$ and alkalinity as well as it is required for the maintenance of cell wall integrity (Hagiwara et al., 2008; Spielvogel et al., 2008). The $\Delta c r z A$ mutant of $A$. fumigatus shows altered temperature, $\mathrm{pH}$ and ion tolerance, defects in germination, polarized growth and asexual development and loss of virulence (Cramer, Jr. et al., 2008; Soriani et al., 2008), and, finally, in A. parasiticus CrzA is required for growth and aflatoxin biosynthesis under calcium stress conditions (Chang, 2008).

To better understand calcium signaling in filamentous fungi, we further investigated cis and trans-regulatory elements acting on CrzA from A. nidulans. Functional, biochemical and cellular localization studies have provided a detailed model of phospho-regulation and nucleocytoplasmic transport of CrzA. We show that both calcium and alkalinity trigger CrzA nuclear localization and activation probably based on a phosphorylation/dephosphorylation process. Like in other fungal models, A. nidulans CrzA is a target for the phosphatase calcineurin. Furthermore, CrzA-inactivation mechanism shares components of the mammalian NFAT and yeast Crzlp pathways, since $A$. nidulans casein kinase I CkiA and A. nidulans Gsk3 $\beta$ homologue are involved in CrzA phosphorylation.

\section{Results}

\section{Calcium, manganese or alkaline ambient $\mathrm{pH}$ stress induce nuclear accumulation of $\mathrm{Crz} A$}

We demonstrated the role of CrzA for tolerance of Aspergillus nidulans to both alkalinity and an elevated extracellular concentration of calcium (Spielvogel et al., 2008), and others also showed this for tolerance to manganese cation (Hagiwara et al., 2008). Calcium, as shown for Crzlp (Cyert, 2003), induces the nuclear accumulation of CrzA ((Spielvogel et al., 2008) and Fig. 1). To determine whether nuclear transport of CrzA is a major regulatory step we analyzed 
1 of cations and different ambient $\mathrm{pH}$ values. Cells of a strain expressing a CrzA-GFP fusion were

2 grown in standard minimal medium without cation or $\mathrm{pH}$ stress, hereafter designated as resting

3 cells, and, as expected, CrzA remained cytoplasmic and excluded from nuclei ((Spielvogel et

4 al., 2008) and Fig. 1). Nuclei were identified using the specific nuclear marker Histone H1

5 tagged with monomeric-cherry fluorescent protein, H1-mCh. Addition of calcium induced

6 nuclear accumulation of CrzA ( $50 \mathrm{mM} \mathrm{CaCl}_{2}$ shown in Fig. 1) and this effect was also observed

7 when the medium was alkalinized using $100 \mathrm{mM} \mathrm{Na}_{2} \mathrm{HPO}_{4}$ as buffering agent (pH 8).

8 Manganese, $\mathrm{Mn}^{2+}$, is a toxic cation for Aspergillus at low concentrations (7.5 mM, (Hagiwara et

9 al., 2008)) and addition of $10 \mathrm{mM} \mathrm{MnCl}_{2}$ also induced the nuclear accumulation of CrzA (Fig.

$101)$.

11 However, CrzA remained preferentially cytoplasmic, as in resting cells, after addition of the 12 divalent cation $\mathrm{Mg}^{2+}\left(200 \mathrm{mM} \mathrm{MgCl}_{2}\right)$ to the medium or after its acidification (using $100 \mathrm{mM}$

$13 \mathrm{NaH}_{2} \mathrm{PO}_{4} ; \mathrm{pH}$ 5.2). A similar effect was observed when an elevated concentration of sodium (1

$14 \mathrm{M} \mathrm{NaCl})$ or potassium $(1 \mathrm{M} \mathrm{KCl})$ was added to the culture. In these later conditions, CrzA-GFP

15 fluorescence aggregated at the cytoplasm but nuclei remained empty, as showed by DAPI

16 staining (Fig. 1). These results strongly support a model in which nuclear transport is a key

17 regulatory point for modulating CrzA transcriptional function. Nuclear accumulation of CrzA is

18 observed in those stress conditions in which the function of this TF is required.

\section{Delimiting functional regions in $\operatorname{CrzA}$.}

A basic deletion analysis has provided with valuable information on the signaling and functional mechanisms acting upon CrzA. Sequence conservation among yeast, Crzlp, and filamentous fungal CrzA homologues is poor and practically concentrates at the DNA binding region (DBD) which comprises three zinc-fingers, two of which belong to the $\mathrm{C}_{2} \mathrm{H}_{2}$ class (Spielvogel et al., 2008). A major difference between Crzlp and CrzA-like proteins is the presence of a conserved C-terminal region after the DBD in CrzA homologues (Supplementary Fig. 1A). Using a expression system based the $g p d A^{\operatorname{mini}}$ promoter, a constitutive but low-expression gpdA promoter (Pantazopoulou and Peñalva, 2009), the functionality of truncated CrzA proteins was analyzed in wild-type and null crzA genetic backgrounds. Expression of GFP-CrzA(1-730), the full-tagged version, complemented calcium and alkaline-pH sensitive phenotypes caused by the null crzA allele (Fig. 2A). Deletion of residues 613-730, the conserved C-terminal region, resulted in a functional truncated CrzA protein. The GFP-CrzA(1-612) protein complemented the null crzA allele for resistance to calcium and alkaline $\mathrm{pH}$ (Fig. 2A). Interestingly, this phenotype was also observed in a strain expressing a truncated CrzA lacking the first 444 amino acids, GFP-CrzA(445-730) (Fig. 2A). In fact, colonies expressing GFP-CrzA(1-612) fusion 
1 grew less healthy than those expressing the full length construct or a truncated GFP-CrzA(445-

2 730) fusion, indicating a functional role for this conserved C-terminal region.

3 As expected fluorescent chimeras lacking the DBD, GFP-CrzA(1-448) and GFP-CrzA(608-

4 730), were not functional (Fig. 2A). None of the previous fusion proteins caused any major

5 distortion in a wild-type $c r z A$ background, however, expression of the zinc-finger region alone

6 reduced colony growth and when in presence of calcium generated multiple sectors emerging

7 from the inoculation point (Fig. 2A, see $\mathrm{Ca}^{2+} / w t$ background sector). As a possible source of

8 loss-of-function mutations in the DBD, we analyzed some of these sectors finding in all cases

9 that either the construct was not expressed or had been deleted from the site of integration

10 within the genome, suggesting a deleterious effect due to constitutive over-expression of this

11 domain (data not shown).

12 To extend the functional analysis of $\mathrm{CrzA}(1-612)$ and $\mathrm{CrzA}(445-730)$, these forms were

13 expressed as unique source of CrzA from its endogenous promoter as C-terminally tagged GFP

14 fusion proteins. As shown above for the N-terminal GFP-tagged CrzA, the CrzA(1-730)-GFP

15 fusion protein is functional (see also (Spielvogel et al., 2008)) and colonial growth of a strain

16 expressing this fusion is indistinguishable of a wild type (Fig. 2B). The $c r z A^{\text {prom }}$-driven

17 expression of CrzA(1-612) also resulted in a functional truncated version but a strain expressing

18 CrzA(445-730) showed some sensitivity to alkaline $\mathrm{pH}$ and no growth under calcium stress

19 conditions (Fig. 2B). Tolerance of A. nidulans to calcium was also tested and a concentration

20 higher than $0.5 \mathrm{M}$ affected colonial growth. The strain expressing the CrzA(1-730)-GFP showed

21 a wild-type phenotype but that expressing CrzA(1-612)-GFP displayed strong sensitivity to

22 concentrations of $\mathrm{CaCl}_{2}$ above $0.5 \mathrm{M}$ (Fig. 2B).

23 Analysis of the cellular distribution of these GFP-chimeras shed light on the location of putative

24 nuclear transport sequences in CrzA (Fig. 2C). Distribution of CrzA(1-612) was comparable to

25 CrzA(1-730), both located mainly at the cytoplasm and excluded from nuclei in resting cells and 26 nuclear under $\mathrm{Ca}^{2+}$ or $\mathrm{pH} 8$ stresses. $\mathrm{CrzA}(1-448)$ holds regulatory regions required to mediate

27 correct nucleocytoplasmic trafficking, although nuclear import was apparently reduced under 28 cation or alkaline-pH stresses. However, CrzA(445-730) was always located in nuclei, in both resting cells and stress conditions. CrzA(445-612), essentially the DBD, also showed nuclear localization independently of ambient conditions. Notably, nuclear accumulation of CrzA(445612) was increased compared to $\mathrm{CrzA}(445-730)$, suggesting that $\mathrm{C}$-terminal region might modulate nuclear import. Nevertheless, CrzA(608-730) seems not to carry any nuclear localization or cytoplasmic retention signal as it showed a dispersed distribution along the cell under any condition assayed. Therefore, calcium and alkaline signaling requires the N-terminal region of CrzA, and this involves the regulation of nucleocytoplasmic transport signals. 
1 Nuclear accumulation of CrzA induced by calcium and alkalinity depends on calcineurin

2 activity.

3

4 A $\triangle c n a A$ strain displays impaired colonial growth with severe morphological defects and sensitivity to an elevated concentration of calcium or to alkalinity (see (Soriani et al., 2008) and Fig. 3A). We focused to determine whether the catalytic subunit of the calcineurin, CnaA, is needed for nucleocytoplasmic transport of CrzA in response to stress. Strain MAD3222 expresses the CrzA-GFP chimera as unique source of this TF in the absence of calcineurin activity $(\triangle c n a A)$. In the absence of stress, CrzA-GFP fluorescence was excluded from nuclei and dispersed through the cytoplasm in both wild-type and null cnaA genetic backgrounds (Fig. 3B). These results suggested that the nuclear export system acting on CrzA was not affected. Nuclear accumulation of CrzA in the wild-type cnaA strain was completed in 5-10 minutes after calcium addition or medium alkalinization whereas in the null cnaA strain the localization of CrzA-GFP remained unaltered after 30 minutes of treatment with either stimulus (Fig. 3B). Thus, the calcineurin activity is responsible for the nuclear accumulation of CrzA in response to $\mathrm{Ca}^{2+}$ and alkaline stress in $A$. nidulans.

$\mathrm{CrzA}$ is a target of calcineurin and different phosphorylated forms are detected under stressing and non-stressing conditions

Regulation of S. cerevisiae Crzlp implies the existence of phosphorylated forms which are detected by their differences in electrophoretic mobility (Stathopoulos-Gerontides et al., 1999). The positive role of calcineurin phosphatase in the nuclear import of CrzA prompted us to investigate the phosphorylation status of this TF under stress and in different mutant backgrounds. Various culture conditions and protein isolation procedures were developed to preserve post-translational modified states of CrzA (see Supplementary Text, and Fig. S2A and S2B). Firstly, the electrophoretic mobility of a CrzA-GFP fusion was followed in protein extracts from resting cells of a wild type and a null cnaA mutant. Fig. 4A shows the reduced mobility of this tagged form of CrzA when using protein extracts from the $\triangle c n a A$ MAD3222 strain compared with the mobility of CrzA-GFP from the wild-type cnaA strain (MAD3021). Low mobility of CrzA was indicative of presence of phosphorylated forms and this was confirmed by inhibiting the activity of calcineurin, using cyclosporine A (CsA), and by performing in vitro dephosphorylation assays. A reduction of CrzA-GFP mobility was observed in protein extracts of a wild type, MAD3021, treated with increasing amounts of CsA (Fig. 4A). Notably, this CsA effect on CrzA mobility was never observed to be as strong as the effect of lacking calcineurin activity (see also Fig. 4B). 
1 When evaluating the in vitro activity of lambda protein phosphatase ( $\lambda$ PP) in protein extracts of 2 a wild type we found that mobility of CrzA-GFP did not change after treatment (Fig. 4B, left).

3 This result and the need of using a specific protein-extraction protocol for $\lambda$ PP assays led us to 4 suppose that those were not the ideal conditions to observe phosphorylated forms of CrzA (see 5 Experimental Procedures and Supplementary Text). The use of CsA would prevent calcineurin 6 activity and, consistently with this inhibitor being added to cultures, mobility of CrzA-GFP was significantly reduced. A $\lambda$ PP assay using protein extracts of a wild type grown with CsA clearly showed an increase in the mobility of CrzA fusion, similar to that observed before without CsA treatment (Fig. 4B, right). The mobility of CrzA-GFP was similar in protein extracts from mycelia of null cnaA strain MAD3222 treated with or without CsA, demonstrating that the effect of CsA in CrzA mobility was in fact through inhibiting the activity of calcineurin (Fig. 4C). In addition, these experiments corroborated the existence of phosphorylated forms of 13 CrzA.

14 To verify that CrzA was a substrate of calcineurin we performed an in vitro dephosphorylation assay using human calcineurin. This experiment also showed an increase in mobility of CrzA similar to that observed for the protein phosphatase of $\lambda$ phage (Fig. 4D). The overall data demonstrates that CrzA is a phospho-protein and a target for calcineurin phosphatase activity. Once demonstrated the presence of phosphorylated forms of CrzA in a wild type background, we analyzed the effect of $\mathrm{Ca}^{2+}$ addition or alkalinization in CrzA mobility (Fig. 4E). Calcium had an immediate effect on increasing the mobility of CrzA (1 min time point, Fig. 4E). Because these protein extracts were obtained in a procedure which preserves CrzA phosphorylation states, it is possible to detect differences in the mobility of CrzA after stress treatment (compare 0 and 1 min time points, Fig. 4E). However, this calcium-derived effect was transient and low-mobility forms of CrzA were detected newly at 5 and 15 min after calcium addition. This effect on CrzA mobility was consistent with the signaling model for other Crz factors in which dephosphorylation induce their nuclear entry (Karababa et al., 2006; Stathopoulos-Gerontides et al., 1999).

Surprisingly, alkalinization had a different effect although increase in the mobility of CrzA was also detected (Fig. 4E). At 1 minute after alkalinization it was possible to detect a mixture of bands rather similar to that observed with calcium at 15 minutes. This pattern of bands varied with time and $30 \mathrm{~min}$ after induction of $\mathrm{pH} 8$ stress it was possible to observe mainly the highest mobility of CrzA (see Fig. 4E and Fig. S2B). Meanwhile nuclear accumulation of CrzA-GFP is observed with both stimuli, calcium and alkalinity (Fig. 1), it is not obvious that a complete dephosphorylated state of CrzA is responsible for its preferential nuclear import, and this has been already noted in other calcineurin signaling and nuclear transport systems (Okamura et al., 
2 Calcineurin-calcium and alkaline stress response elements in CrzA locate at the N-terminal

3 region.

5 The $\operatorname{CrzA}(1-612)$ truncated protein holds the regulatory regions required to mediate 6 nucleocytoplasmic trafficking in response to calcium stress and alkalinity (Fig. 2C). The need of 7 calcineurin signaling in this transport process was evaluated by expressing the GFP-CrzA(18 612) fusion, driven by the $g p d A^{m i n i}$-promoter, in the $\Delta c n a A$ mutant. In the absence of calcineurin 9 activity, the addition of calcium or medium alkalinization did not promote nuclear import of 10 GFP-CrzA(1-612), and this was also observed for the, $g p d A^{\min i}$-driven, full GFP-CrzA fusion 11 (Fig. 5A). Accordingly with the non-regulated nuclear localization shown by the GFP12 CrzA(445-730) truncated form, the absence of calcineurin did not alter its nuclear accumulation 13 at any growth condition (Fig. 5A). These results suggested that residues lying within 1-612 14 region might be under regulation by phosphorylation-dephosphorylation. $\lambda$ PP assays confirmed 15 that CrzA(1-612) was phosphorylated in a null cnaA mutant, whereas CrzA(445-730) was not 16 (Fig. 5B). Thus calcineurin regulates signaling (nucleocytoplasmic trafficking) of CrzA in 17 response to $\mathrm{Ca}^{2+}$ and $\mathrm{pH} 8$ and this must require, at least, a docking domain located into the first 445 amino acids of this TF. In addition, constitutive expression of these full or truncated-CrzA forms did not suppress phenotypes derived of $\triangle c n a A$ allele such as sensitivity to calcium or alkaline-pH stress, and defects in the colony morphology (Fig. 5C).

\section{Identification of a CnaA docking domain in CrzA}

The catalytic subunit of calcineurin recognizes in its substrates a short motif, denoted as calcineurin-docking domain (CDD), which conforms the consensus sequence PxIxIT defined in NFAT transcription factors (Fig. 6A). A possible dual calcineurin-docking site was predicted between amino acids Q294 and H316 (Spielvogel et al., 2008) (Fig. 6B). To study the role of this putative CDD a mutant version of GFP-tagged CrzA lacking this region, designated as CDD1 (Fig. 6B), was generated by gene replacement at the crzA locus. Transformants expressing $\mathrm{CrzA}^{\triangle \mathrm{CDD} 1}$-GFP as unique source of CrzA displayed a similar phenotype to that observed for the $c r z A$ wild-type strain with respect to calcium or alkaline-pH tolerance (Fig. $6 \mathrm{C}$ ). In agreement with this observation, $\mathrm{CrzA}^{\Delta \mathrm{CDD1}}$-GFP accumulated in nuclei after calcium or alkaline-pH stress (Fig. 6D), indicating that signaling of this mutant protein occurs as in the native CrzA protein.

35 An alternative CDD of PxIxIT type was found between amino acids M356 and A371, a 36 PSINVE motif designated as CDD2, sharing strong similarity to the low-affinity Crz1p 
1 calcineurin-binding sequence PVIAVN (Roy et al., 2007). This PSINVE motif is conserved 2 among CrzA homologues of filamentous fungi (Fig. 5A and B). A strain expressing a mutant 3 version of CrzA lacking CDD2, $\mathrm{CrzA}^{\triangle \mathrm{CDD} 2}$-GFP, was obtained as above for site CDD1. In 4 contrast to the wild type-like localization of $\mathrm{CrzA}^{\Delta \mathrm{CDD} 1}-\mathrm{GFP}$, the mutant $\mathrm{CrzA}^{\Delta \mathrm{CDD} 2}-\mathrm{GFP}$ 5 remained cytoplasmic after medium alkalinization or calcium addition (Fig. 5D). The 6 localization of mutant $\mathrm{CrzA}^{\triangle \mathrm{CDD} 2}$-GFP resembled that of CrzA-GFP in the absence of 7 calcineurin activity.

8 However, a strain expressing $\mathrm{CrzA}^{\triangle \mathrm{CDD} 2}$-GFP protein still displayed mild sensitivity to elevated

9 levels of calcium or alkalinity. To study a possible synergic effect of CDD1 site in this stress 10 tolerance a mutant strain expressing a CrzA-GFP fusion protein lacking both CDD sites was 11 generated, $\mathrm{CrzA}^{\Delta \mathrm{CDD1}+2}$-GFP. Localization of $\mathrm{CrzA}^{\triangle \mathrm{CDD1}+2}-\mathrm{GFP}$ was indistinguishable of $12 \mathrm{CrzA}^{\Delta \mathrm{CDD} 2}$-GFP protein and both $\triangle \mathrm{CDD} 2$ - and $\triangle \mathrm{CDD} 1+2-\mathrm{CrzA}$-mutant strains displayed 13 similar tolerances to calcium and alkalinity (Fig. 6C). An explanation for this contradictory 14 phenotype was obtained when analyzing the electrophoretic mobility of mutant $\triangle \mathrm{CDD} 1+2-$ 15 CrzA. Addition of calcineurin inhibitor CsA reduced the mobility of $\mathrm{CrzA}^{\triangle \mathrm{CDD1}+2}$-GFP (Fig. 6E) 16 and $\lambda$ PP assays of protein extracts from mycelia treated or not with CsA showed CrzA ${ }^{\triangle \mathrm{CDD} 1+2}$ 17 GFP was phosphorylated differently in both conditions (Fig. 6F). Calcium but not medium 18 alkalinization increased the mobility of this mutant CrzA protein as observed before for the full 19 CrzA-GFP fusion, although a delay in this response was observed (Fig. 6E). These results 20 indicated that this mutant CrzA protein could be subjected to a 21 phosphorylation/dephosphorylation process mediated by calcineurin by recognizing alternative sites such as LxVP motif (Rodriguez et al., 2009). However, a scanning of this CnaA binding motif in CrzA sequence was unsuccessful suggesting either the presence of novel CDD in CrzA or a direct action of this phosphatase without docking. Finally to confirm that CDD2 is a key element on CrzA-calcineurin interaction a pull down experiment was performed using bacterially expressed GST-CrzA fusions either in its wild-type sequence or lacking the CDD2 motif, and protein extracts from strain MAD4366 expressing a CnaA-GFP fusion. A CrzA-CnaA interaction was detected when using the wild-type version of GST-CrzA but not with the $\mathrm{CrzA}^{\triangle \mathrm{CDD} 2}$ (Fig. 6G), thus we propose CDD2 as the most important site for calcineurin signaling in CrzA.

Mutations in casein kinase I, CkiA, affect CrzA phosphorylation but not nucleocytoplasmic transport. 
1 2012). Three partial loss-of-function mutants in ckiA, alleles ckiA2, ckiA1919 and ckiA102, and 2 a conditional, thiamine-dependent, null allele thi $A^{p}:: c k i A$ (kindly provided by Prof. Scazzocchio 3 and Dr. Sotiris), were analyzed for their potential effects on CrzA phosphorylation.

4 Figure 7A and Fig. S3 show the colonial growth of ckiA mutant strains expressing the CrzA5 GFP chimera, as the unique source of CrzA, on different media conditions. There are no visible 6 effects of excess of calcium or alkalinity in the colonial growth of these ckiA mutants (Fig. 7A 7 and Fig. S3) but they showed the expected sensitivity or reduced growth due to defects in amino 8 acid transport (shown for arginine in Fig. 7A, and see also (Apostolaki et al., 2012)). A triple 9 mutant $c k i A 2$, null cnaA, crzA-gfp strain was also constructed and displayed a similar phenotype to that of $\triangle c n a A, c r z A-g f p$, strain except for reduced growth in medium containing arginine as main nitrogen source due to the presence of $c k i A 2$ mutation.

12 We analyzed the localization of CrzA-GFP in these partial loss-of-function ckiA backgrounds 13 and it was similar to that described above for this fusion protein in control strain MAD3021, 14 displaying nuclear exclusion in the absence of stress and nuclear accumulation after 15 alkalinization or calcium addition (Fig. 7B). Importantly, CrzA-GFP also showed the 16 requirement of calcineurin activity to be imported in the nucleus in the ckiA2 background (Fig. 7B). These results suggested that CrzA was still subjected to phosphorylation and to verify this hypothesis protein extracts ckiA mutant strains were analyzed. The mobility of CrzA-GFP in each of the ckiA partial loss-of-function mutants was higher compared to that found in extracts from the wild-type ckiA strain (Fig. 7C). In particular, ckiA102 and ckiA2 showed higher mobility of CrzA in contrast to ckiA1919 which is a moderate loss-of-function allele (Apostolaki et al., 2012). Mobility of CrzA was reduced in these mutant backgrounds when CsA was added, however this mobility was not exactly that found for the CrzA form described under the same conditions in a wild type (compare Fig. 4 and 7C), suggesting a reduction on the phosphorylation levels of CrzA in the absence of full CkiA activity. When analyzing the conditional null ckiA strain we found that mobility of CrzA was similar to that observed in a ckiA2, the strongest loss-of-function mutant, either in the absence or presence of calcineurin inhibitor (Fig. S3). This effect was also observed in a ckiA2, $\triangle c n a A$ mutant background where the electrophoretic mobility of CrzA was higher than that of CrzA in a null cnaA background (Fig. 7C). These results evidenced a role of CkiA casein-kinase in the phosphorylation level of CrzA, but functionally, this might have a minor impact because it is not affecting either the regulation of nuclear import and/or export mechanisms for CrzA, or an adequate response to calcium and alkaline-pH stress by this transcription factor. 
2 Glycogen-synthase kinase 3- $\beta$ (GSK3 $\beta$ ) phosphorylates NFAT factors (Beals et al., 1997), 3 being an alternative mechanism to casein kinase I for CrzA phosphorylation. A motif scan of 4 the CrzA sequence, using NetPhosk 1.0 on-line resources 5 (www.cbs.dtu.dk/services/NetPhosK/), revealed the presence of numerous putative target sites 6 for a GSK3 $\beta$-like kinase activity, mostly located at the N-terminal region with respect to the 7 DBD (Fig. 8A).

8 We identified the locus coding for $A$. nidulans GSK3 $\beta$ homologue, AN6508, using BlastN and 9 BlastP searches and generated a null An6508-GSK3 $\beta, \Delta g s k A$ strain. This putative kinase, in 10 contrast to $c k i A$, is not essential but the mutant $g s k A$ strain displays a severe defect of colonial 11 growth and poor conidiation (Fig. 8B, these results are consistent with those presented recently 12 in a systematic functional analysis of $A$. nidulans kinome (De Souza et al., 2013)). Despite the strong morphological defects, cellular localization of CrzA-GFP was not altered and exhibited a wild type-like distribution in the absence of $\mathrm{Ca}^{2+}$ or $\mathrm{pH} 8$ stresses (Fig. 8C). Nuclei of $\Delta g s k A$ resting cells were altered in their cellular distribution and size, but this did not affect the preferential cytoplasmic localization of CrzA-GFP (Fig. 8C). Calcium induced nuclear entry of the fusion protein similarly to other $\mathrm{Ca}^{2+}$-stimulation experiments. However alkaline-pH stress did not cause the same effect in the localization of CrzA. After alkalinization nuclear exclusion of CrzA-GFP was lost. Although nucleoplasm became fluorescent, accumulation of CrzA-GFP was not immediately observed (see magnification of a selected nucleus, Fig. 8C), or even after 30 min treatment (not shown), indicating that GskA activity is a requisite to activate the nuclear import mechanism of CrzA under alkaline-pH stress.

Phosphorylation levels of CrzA are reduced in the $\Delta g s k A$ mutant. Mobility of CrzA in $\Delta g s k A$ was higher than in the wild type RC (Fig. 8D), being this effect shared with mutants in CkiA activity (see above, Fig. 7C and Fig. S2C). Treatment of $\Delta g s k A$ cells with CsA reduced CrzA mobility but not to the level found in the wild type (Fig. 8D) or ckiA mutants (Fig. S2C), suggesting a very low level of phosphorylation of CrzA in the $\Delta g s k A$, which was confirmed by $\lambda$ PP assays (Fig. 8E). In fact, the mobility of CrzA in the null gskA background was similar to that of a completely dephosphorylated CrzA form (Fig. 8E and Fig. S2C). Nevertheless, CrzA is susceptible to be phosphorylated in the absence of GskA activity as showed the reduction in CrzA mobility after CsA treatment and further dephosphorylation assays using $\lambda$ PP (Fig. 8E). Interestingly, a reduction in electrophoretic mobility of CrzA in the $\Delta g s k A$ mutant was also detected after addition of calcium or medium alkalinization, indicating that both stimuli are inducing a modification in this TF in the absence of GskA kinase (Fig. 8F). The overall results clearly support a fundamental role of GSK3 $\beta$ homologue in proper signaling of 36 CrzA. 
5 Signaling of calcineurin-responsive zinc-finger $(\mathrm{Crz})$ proteins has been studied in detail only in 6 S. cerevisiae (Cyert, 2003). Here we demonstrate that signaling mechanisms acting on a 7 filamentous fungal Crz, CrzA of Aspergillus nidulans, are conserved with yeast Crzlp and 8 mammalian NFATc transcription factors. CrzA is a phospho-protein that is dephosphorylated in response to a limited number of ambient stress factors through the calcineurin phosphatase. Essentially, calcineurin activity is required for CrzA to migrate into nuclei (see regulatory model in Fig. 9). Activities of at least two kinases, the casein kinase I homologue, CkiA, and glycogen-synthase- $3 \beta$ kinase homologue, GskA, are involved in CrzA phosphorylation. We visualized a wide range of electrophoretic mobility for CrzA, however it was not possible to correlate phosphorylation level with cellular localization. In fact, activity of GskA is required for nuclear accumulation of CrzA at ambient alkaline $\mathrm{pH}$ but not for calcium stress. Thus a precise balance of phosphorylation/dephosphorylation must be required to modulate nucleocytoplasmic trafficking of CrzA in response to a limited number of extracellular signals.

\section{Nuclear entry of $\mathrm{CrzA}$, a major regulatory step in its signaling.}

A null $\mathrm{crz} A$ strain displays sensitivity to elevated concentrations of extracellular calcium (above $10 \mathrm{mM}$ ), manganese (above $5 \mathrm{mM}$ ) and alkaline $\mathrm{pH}$ (above $\mathrm{pH} 7.5$ ), suggesting that the transcriptional function of CrzA is required for tolerance to these abiotic stresses (Hagiwara et al., 2008;Spielvogel et al., 2008). In agreement with this hypothesis we observed that CrzA accumulated in nuclei when cells are exposed to those stresses. In resting cells, CrzA as other homologues in fungi exhibits a cytoplasmic distribution being excluded from nuclei (Karababa et al., 2006;Lev et al., 2012; Stathopoulos-Gerontides et al., 1999), and here we show that this localization of CrzA is maintained in those stress conditions which do not require its function (Fig. 1). An excess of monovalent cations such as sodium or potassium and the divalent cation magnesium do not induce nuclear entry of CrzA. Interestingly, whereas magnesium or medium acidification do not alter the cytoplasmic distribution of CrzA, addition of elevated concentrations of $\mathrm{Na}^{+}$or $\mathrm{K}^{+}$cations induce the presence of fluorescent aggregates in the cytoplasm as those recently observed in Cryptococcus neoformans upon salt-stress (Lev et al., 2012). The nature of those aggregates has not been studied yet in A. nidulans, but its presence indicates the existence of a common fungal mechanism acting on CrzA homologues to prevent nuclear entry under salt stress.

36 Thus, in a specific way, calcium, manganese and alkalinity must stimulate the nuclear import system. Our functional analysis of CrzA indicates the presence of a NES within the first 445 
1 amino acids and an NLS in its proximities as shows the localization of $\mathrm{CrzA}(1-448)$ truncated

2 form. However, we detect a second NLS into CrzA(445-612) fragment, most probably the

3 immediacy or into the DBD. A similar number and distribution of nuclear transport signals were

4 described in Crzlp (Boustany and Cyert, 2002;Polizotto and Cyert, 2001), but functional

5 conservation extends to their regulation by stress. As in Crz1p, a CrzA fragment containing both

6 N-terminal NLS and NES, CrzA(1-448), is regulated by calcium or alkalinity, and a fragment

7 containing essentially the $\mathrm{DBD}, \mathrm{CrzA}(445-612)$, is permanently located in the nucleus

8 independently of calcineurin signaling. The absence of sequence conservation between Crzlp

9 and CrzA prevented the identification of both NES and NLS in the N-terminal region, however

10 the strong sequence conservation at the zinc-finger region clearly identifies Crz1p-NLS2,

$11{ }^{612} \mathrm{RKR}^{615}$, into CrzA DBD. Finally, it was shown that a null $n m d 5$ strain displayed the same

12 phenotype as a null $\mathrm{crzl}$ strain demonstrating the role of this karyopherin in nuclear import of

13 Crz1p (Polizotto and Cyert, 2001). During our analysis of nuclear transport pathways in $A$.

14 nidulans was stated the absence of conservation of such phenotype for a null kapD strain, being

15 KapD the putative homologue for Nmd5, and for any other viable null mutant in the transport

16 system (Markina-Inarrairaegui et al., 2011). Currently we are identifying and analyzing these

17 transport signals and possible transporters involved.

\section{Calcineurin activity and post-translationally modified states of CrzA.}

20 In this work we demonstrate for the first time in filamentous fungi a role of calcineurin in calcium and alkaline-pH signaling of CrzA. Calcineurin promotes CrzA dephosphorylation and subsequently this induces its nuclear accumulation. As observed for Crzlp (StathopoulosGerontides et al., 1999), the absence of calcineurin activity restricts CrzA localization to the cytoplasm and the highest level of phosphorylation is observed as the lowest electrophoretic mobility of CrzA. It is interesting to note that such low mobility of CrzA in a $\triangle c n a A$ background was not modified by any external stimulus demonstrating the essential role of calcineurin in this regulatory system of $A$. nidulans. In a previous work (Spielvogel et al., 2008), we predicted a double calcineurin docking domain in CrzA, CDD1, not conforming the PxIxIT consensus but similar to the PIISIQ motif identified in Crzlp (Matheos et al., 1997). However our functional study of double-site CDD1 showed that, although conserved among CrzA homologues, removal of these putative calcineurin binding sequences did not affect signaling of CrzA. In contrast, an alternative site, PSINVE, was found downstream of CDD1 and to be necessary for nuclear translocation of CrzA in response to both calcium and alkaline $\mathrm{pH}$. This conserved putative calcineurin site at CDD2 is similar to a low affinity site for calcineurin studied in Crzlp, PVIAVN (Roy et al., 2007). An in vitro interaction experiment has proven the important role of CDD2 in the interaction between

37 CrzA and CnaA. However, deletion of either CDD2 alone or in combination with CDD1 did not 
1 prevent growth of the mutant strain in the presence of calcium or alkaline $\mathrm{pH}$. This phenotype

2 was anticipated from a loss-of-signaling transduction between active calcineurin and CrzA. The

3 effect of calcineurin-inhibitor $\mathrm{CsA}$ on $\mathrm{CrzA}^{\Delta \mathrm{CCD} 1+2}$ mobility suggested the presence of either

4 residual calcineurin activity or additional, alternative, CDDs. Absence of LxVP motifs

5 (Rodriguez et al., 2009) suggests that if alternative CDDs are present in CrzA, these might be

6 completely unrelated to those defined previously. Detailed experimental work will be required

7 to determine the exact number of CDD sites, however we propose CDD2 as the most important

8 site for calcineurin signaling in CrzA.

9 Demonstration of different phosphorylation levels in CrzA has been possible due to an

10 alternative protocol for protein isolation which prevented, most probably, unspecific activation

11 by calcineurin during the process by reducing manipulation time. In these conditions we

12 visualized five different phosphorylation stages, ranging from forms detected in the absence of

13 calcineurin activity, highest phosphorylation level, to those found after calcium treatment, the

14 lowest (see Fig. S2B and Supplementary Text). In Candida albicans, different phosphorylation

15 steps of CRZ1 have been described after mutant, drug treatments and calcium-addition studies

16 (Karababa et al., 2006). Under non-stressing conditions, mobility of CrzA suggests that is

17 partially phosphorylated and truncated forms indicate that most of target residues locate in the

18 N-terminal region 1-445. The presence of a conserved serine-rich region (SRR) is found

19 between residues 233 and 352 in CrzA, some of which overlap with CDD1 (Fig. 6). Although

20 amino acid sequence is not highly conserved amongst yeast and filamentous fungal $\mathrm{Crz} 1 \mathrm{p} / \mathrm{CrzA}$

21 orthologues, residues targeted for phosphorylation, SRR, locate in the proximities of nuclear

22 transport signals and calcineurin binding sites (Karababa et al., 2006; Stathopoulos-Gerontides

23 et al., 1999).

\section{Kinases involved in CrzA phosphorylation}

26 Here we identify two phosphorylation mechanisms acting on CrzA. The essential casein kinase I, CkiA (Apostolaki et al., 2012), regulates the phosphorylation state of CrzA, as does Hrr25p in S. cerevisiae for Crz1 (Kafadar et al., 2003). All three available partial loss-of-function mutations in CkiA and its conditional null allele modify CrzA phosphorylation levels. In mutant ckiA resting cells CrzA remains cytoplasmic and calcineurin activity was still essential for calcium or $\mathrm{pH} 8$-induced nuclear import suggesting a minor role of CkiA in CrzA signaling. A predicted CK1 phosphorylation site was found at serine 173, however this and flanking residues are not conserved among CrzA homologues (Spielvogel et al., 2008).

Gsk3 $\beta$ kinases recognize a short motif in their substrates, $\mathrm{S} / \mathrm{TxxxS} / \mathrm{T}$, where the last residue is already phosphorylated (Fiol et al., 1987). The presence of eighteen putative target sites in CrzA for a Gsk3 $\beta$-like kinase prompted us to study its homologue in $A$. nidulans. This kinase has not 
1 been studied in-depth in filamentous fungi. Gsk3 $\beta$ homologue in $S$. cerevisiae was designated as

2 RIM11, which also relates this kinase with the RIM101/PacC pathway (Mitchell and Bowdish,

3 1992). Gsk3 $\beta$ homologue in A. nidulans is coded by locus An6508 designated here as gskA. We

4 show that this is not an essential kinase, however its absence cause major morphological defects

5 which are greater in minimal than in complete medium (see also (De Souza et al., 2013)). In the

6 absence of GskA activity CrzA is found in a low phosphorylation state, even when the

7 calcineurin inhibitor was added, indicating a major role of this kinase in CrzA post-translational

8 modification. However, CrzA is excluded from nuclei in null gskA resting cells, thus GskA

9 activity is not required for the nuclear export of CrzA. In the null gskA background, calcium

10 induces nuclear accumulation of CrzA but alkalinity seems preferentially affect the nuclear

11 export system as nuclear exclusion of CrzA is lost. These changes in localization of CrzA are in

12 contrast with an effect of calcium or alkaline $\mathrm{pH}$ in reducing its electrophoretic mobility,

13 indicating the existence of a phosphorylation step in those conditions. CrzA mobility is different

14 between null gskA or ckiA mutants, providing evidence of dissimilar modes of signaling. The

15 overall of these results indicate that GskA is a principal component of CrzA signaling but state

16 that other kinases, possibly activated by calcium or alkalinity, might be acting on CrzA.

17 Participation of stress-induced activated kinases in Crz or NFAT signaling processes have been

18 proposed. In this respect, would be interesting to determine whether cAMP-dependent protein

19 kinase A, PKA, plays a role in post-translational modification CrzA because, Crzlp and NFAT

20 are phosphorylated by PKA, being in the latter that a primary modification by PKA anticipates

21 GSK3 $\beta$-dependent modifications (Kafadar and Cyert, 2004;Sheridan et al., 2002) and a coordinated regulatory mechanism between PKA and RIM11 has been shown for Ime1, the key transcriptional activator of meiosis-specific genes in S. cerevisiae (Rubin-Bejerano et al., 2004).

\section{Integration of calcium and alkaline pH signals}

A regulatory model for CrzA is pictured in Fig. 9. In resting cells equilibrium between modified forms of CrzA is predicted. Antagonistic activities of calcineurin and kinases, at least CkiA and GskA, may regulate the final state of CrzA phosphorylation, and this is a common mechanism among all calcineurin-dependent regulatory systems, including now a filamentous fungus. When no stimulus is present, it is expected that kinase activities predominate on CrzA. CkiA, GskA and other kinases, as those cited above, might be acting alone or in a concerted manner to regulate CrzA.

33 An elevation of cytoplasmic calcium due to an elevated extracellular concentration of this cation activates the calmodulin/calcineurin signaling pathway in a similar way to what has been described in other organisms, and we show here that CrzA is one of its final targets in $A$. nidulans. In S. cerevisiae calcineurin acts on different effectors and subsequent pathways (see 
1 visible in $A$. nidulans, as noted before (Soriani et al., 2008), $\Delta c r z A$ and $\triangle c n a A$ strains do not 2 display identical sensitive phenotypes to either calcium or alkaline $\mathrm{pH}$ (see Fig. 6) and a $\triangle c n a A$ 3 strain exhibits strong mophological defects not displayed by the $\triangle c r z A$ mutant.

4 Alkaline $\mathrm{pH}$ signaling remains still obscure. Elevation of intracellular calcium levels upon stress 5 seems to be a general physiological response for yeasts and mammals (for a review see 6 (Aramburu et al., 2000)), and recently, an alkaline pH-dependent burst of intracellular calcium 7 has been reported in C. albicans (Wang et al., 2011). Hypothetically, alkaline $\mathrm{pH}$ would cause a 8 release of internal calcium stores and induce a similar response to that proposed for calcium 9 stress. Additionally, through other mechanisms alkalinity could act on the phosphorylation step.

10 It is noticeably that alkalinization do not promote a strong dephosphorylation effect in CrzA, 11 although nuclear transport seems to be equally activated.

12 In $A$. nidulans tolerance to alkalinity requires the activity of at least three major regulators, 13 CrzA, SltA and PacC. Here we show that signaling of CrzA to alkaline $\mathrm{pH}$ or calcium does not 14 require a proteolytic processing step as for PacC. The presence of a conserved YPxL motif, as 15 those recognized by PalA in PacC (Vincent et al., 2003), at the C-terminal region of CrzA-like 16 factors suggested a possible relationship of CrzA with the Pal pathway. However there is no 17 evidence of such a Pal/CrzA crosstalk since any interaction was detected between CrzA and 18 PalA (Fig. S1B). We have not found any direct relationship, at this stage, between calcineurin 19 and $\mathrm{PacC}$ or SltA. Thus alkaline $\mathrm{pH}$ adaptation may occur through parallel signaling pathways but common target genes regulated by these transcription factors, as has been suggested before in (Spielvogel et al., 2008), or in C. albicans for CRZ1/2 and RIM101 (Kullas et al., 2007).

\section{Experimental Procedures}

\section{Strains and media}

Aspergillus nidulans strains used in the present study are listed in Table 1. Escherichia coli strain DH5 $\alpha$ (GibcoBRL) was used for standard DNA cloning, plasmid manipulation or propagation, and GST fusion expression. General molecular techniques followed (Sambrook et al., 1989). A. nidulans strains were cultivated in appropriately supplemented standard minimal medium (MMA) and complete medium (CMA) for Aspergillus as described previously (Cove, 1966). Colony growth was studied by inoculating conidiospores on solid MMA supplemented with $50 \mathrm{mM} \mathrm{CaCl}_{2}$ or $0.1 \mathrm{M} \mathrm{Na}_{2} \mathrm{HPO}_{4}$ to generate alkaline $\mathrm{pH}(\mathrm{pH} 8)$ and incubated at $37^{\circ} \mathrm{C}$ for 2 days.

34 For total protein extractions, strains were cultivated in fermentation media for 18 hours at $37^{\circ} \mathrm{C}$ under agitation, essentially as described in (Orejas et al., 1995). When indicated, calcium or 
1 growing culture and further incubated at $37^{\circ} \mathrm{C}$ for time periods from 1 to 30 minutes, as

2 indicated in the text, before mycelium collection. Calcineurin activity was inhibited in the

3 presence of the inmunosuppresant Cyclosporin A (CsA). CsA (Sigma-Aldrich) stock was

4 prepared in dimethyl sulfoxide and added to the fermentation culture at final concentration of 50

$5 \mu \mathrm{M}$ and incubated for 30 minutes at $37^{\circ} \mathrm{C}$, otherwise indicated.

6

\section{Construction of strains}

8 Gene replacements, favored by the presence of null nkuA mutations, followed protocols 9 described in (Markina-Inarrairaegui et al., 2011;Nayak et al., 2006). Strains obtained by 10 transformation using either linear-DNA fragments or plasmids followed the protocol described 11 in (Tilburn et al., 1983). As selectable markers different genes from Aspergillus fumigatus were 12 used, such as $p y r G$, riboB or pyroA. Pyrimidine, riboflavin or pyridoxine prototrophs were 13 selected and homokaryotic transformants carrying single-copy integration events were identified 14 by Southern blotting (Sambrook et al., 1989). Mutant strains carrying GFP-tagged CrzA 15 cassettes with either $\triangle c n a A$ and/or ckiA mutant alleles were obtained by meiotic recombination 16 following standard genetic procedures in this fungus. Tagging of CnaA with GFP followed the 17 procedures indicated in (Markina-Inarrairaegui et al., 2011;Nayak et al., 2006). A 18 transformation cassette was generated by fusion PCR procedures using oligonucleotides listed in Table S1, and transformed into recipient strain MAD1739. Among $p y r G^{+}$transformants those showing a double recombination event at cnaA locus were selected and tested for expression of the CnaA-GFP chimera, MAD4366 was used in this work.

\section{Deletion, GFP-tagging and directed mutagenesis of crzA locus}

24 Two different null $c r z A$ strains were used in this work, $\triangle c r z A: \because p y r 4^{N c}$ and $\triangle c r z A: \because r i b o B^{A f}$ (MAD2449 and MAD3709). Strain MAD2449 was constructed by replacing the crzA coding sequence with the pyr4 gene from Neurospora crassa as described for strain BER02 (see (Spielvogel et al., 2008)). For the construction of MAD3709 strain the wild-type $c r z A$ locus was replaced by the $A$. fumigatus riboB, riboB ${ }^{A f}$, following the procedure described in (MarkinaInarrairaegui et al., 2011). A replacement cassette was generated by fusion to the $r i b o B^{\mathrm{Af}}$ gene of $1.5 \mathrm{~kb}$ PCR-amplified fragments corresponding to the 5' and 3' UTRs of AN5726 locus, using oligonucleotides pairs $\mathrm{CrzA}(1) / \mathrm{CrzA}(2)$ and $\mathrm{CrzA}(3) / \mathrm{CrzA}(4)$, respectively. The $A$.

32 fumigatus riboB cassette was amplified from plasmid p1830, which contains a $2.2 \mathrm{~kb}$ insert of 33 the genomic sequence corresponding to locus Afulg13300, using specific primers $34\left(\mathrm{CrzA}\left(2^{\prime}\right) / \mathrm{CrzA}\left(3^{\prime}\right)\right)$. Oligonucleotides for this and subsequent sections are listed in Table S1.

35 A strain expressing a C-terminally GFP-tagged CrzA was generated by (Spielvogel et al., 36 2008). Using primer pair $\mathrm{CrzA}(1)$ and $\mathrm{CrzA}(4)$ a fragment comprising

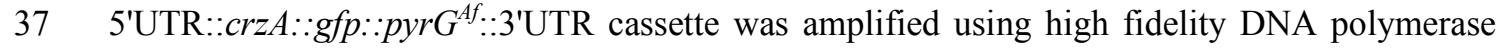


1 PrimeStar (Takara) and cloned into plasmid pGEM-T-Easy (Promega) to give plasmid pGEM-

2 T-CrzA-GFP. The absence of mutations in the $c r z A: \because g f p / p y r G^{A f}$ construct was verified by

3 sequencing and subsequently used as template in further PCR amplifications. Strain MAD4641

4 co-expressing CrzA-GFP and histone H1 tagged with monomeric cherry fluorescent protein was

5 obtained after transformation of MAD2446 with the tagged $c r z A$-transformation cassette.

6 Strains expressing truncated forms of the C-terminally GFP-tagged CrzA, CrzA(1-612) and

$7 \mathrm{CrzA}(445-730)$, were generated by replacement of $\triangle c r z A:: r i b o B^{A f}$ allele in MAD3709 strain,

8 using 5 'UTR::crzA(1-612)::gfp/pyrG $G^{A f}:: 3^{\prime} \mathrm{UTR}$ and 5'UTR::crzA(445-730)::gfp/pyrG $G^{A f}:: 3^{\prime} \mathrm{UTR}$

9 cassettes. Both DNA cassettes were generated in a two-way PCR by fusion of fragments

10 amplified from pGEM-T-CrzA-GFP using primer pairs CrzA(1)/CrzA(1-612)rv and CrzA(1-

11 612)fw/CrzA(4) for the $c r z A(1-612): \because g f p$, construct, and primer pairs $\operatorname{CrzA}(1) / \operatorname{CrzA}(2)$ and

$12 \mathrm{CrzA}(445-730) \mathrm{fw} / \mathrm{CrzA}(4)$ for the $\operatorname{crzA}(445-730):: g f p$, construct.

13 Strains expressing $\mathrm{CrzA}^{\Delta \mathrm{CDD} 1}-\mathrm{GFP}, \mathrm{CrzA}^{\triangle \mathrm{CDD} 2}$-GFP or $\mathrm{CrzA}^{\Delta \mathrm{CDD} 1+2}-\mathrm{GFP}$ mutant versions were 14 generated by replacement of $\triangle c r z A:: r i b o B^{A f}$ locus using PCR-mutagenized

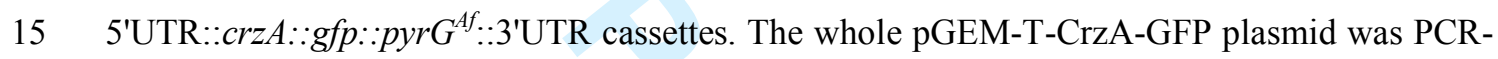
16 amplified using mutant primer pairs to specifically delete either CDD1 or CDD2 site. CDD1 site 17 was deleted using primer pair $\triangle \mathrm{CDD} 1-\mathrm{fw} / \triangle \mathrm{CDD} 1-\mathrm{rv}$. CDD2 site was deleted using primer 18 pair $\triangle \mathrm{CDD} 2-\mathrm{fw} / \Delta \mathrm{CDD} 2-\mathrm{rv}$. The PCR product was digested with $D p n I$ before transformation 19 in E. coli DH5 $\alpha$ to select mutagenized plasmids. Plasmid carrying the double deletion of CDD1 20 and CDD2, pGEM-T- $\mathrm{CrzA}^{\Delta \mathrm{CDD1}+2}$-GFP, was generated as described above using pGEM-T-

$21 \mathrm{CrzA}^{\Delta \mathrm{CDD} 1}$-GFP as template and mutant primers $\triangle \mathrm{CDD} 2$-fw and $\triangle \mathrm{CDD} 2$-rv. In each case 22 sequencing confirmed the proper deletion of the CCDs before amplification and use in 23 transformation. Recipient strain for these constructs was MAD3709.

24 When required, the riboB2 allele was replaced by the wild-type riboB by transformation with a $251.3 \mathrm{~kb}$ PCR fragment amplified from a wild-type strain using primers RiboB ${ }^{\mathrm{AN} 0670}$ up and RiboB ${ }^{\mathrm{AN} 0670}$ down, and further selection of transformants for riboflavin prototrophy.

Expression of GFP-CrzA fusion driven by the gpdA-mini promoter

29 Plasmid p1660 is the recipient for all constructs and holds the $g p d A^{\min i}$ promoter and a truncated pyro $A$ used as selection marker for directed integration at pyroA4 mutant locus (Pantazoupoulou and Penalva, 2009). Fragments or complete CDS of CrzA were obtained by PCR amplification of crzA cDNA cloned in plasmid pGEM-T-CrzAcDNA (Spielvogel et al., 2008). Specific primer pairs carrying $N c o$ I or EcoRI sites were used for amplification of different CrzA regions studied in this work. These fragments were cloned into plasmid pGEM-T-Easy (Promega), sequenced to avoid undesirable mutations due to PCR polymerase and excised using $N c o$ I and 
1 the fragment coding for GFP, each derivative p1660-CrzA plasmid was digested with NcoI and 2 an NcoI-NcoI fragment from plasmid p1155 (Mingot et al., 2001) was inserted providing with $3 g p d A^{\text {mini }}:$ GFP::CrzA-complete/truncated plasmid collection. Fidelity and the correct orientation 4 of the inserts were verified by DNA sequencing. Plasmids were targeted in single copy to the 5 pyroA locus as described previously (Pantazopoulou and Peñalva, 2009) using strains 6 MAD1425 and MAD2449.

Expression of GST-CrzA fusions in bacteria and pull down assays.

9 Two recombinant vectors of original pGEX-2T (GE Healthcare) expressing either wild type or $\triangle \mathrm{CDD} 2 \mathrm{CrzA}$ versions fused to Glutathione S-transferase (GST) were constructed. Firstly plasmid pGEX-CrzA was generated by introducing the cDNA of $c r z A$ coding region in frame with GST coding gene. CrzA coding fragment was generated by PCR and NcoI and EcoRI restriction sites were included at each end of the fragment using specific oligonucleotides (see Table S1). This fragment, after $N c o$ I and EcoRI digestion, was inserted into an already modified version of pGEX-2T containing an NcoI between BamHI and EcoRI at the polycloning site of the original plasmid. pGEX-CrzA ${ }^{\Delta \mathrm{CDD} 2}$ was generated by mutagenic PCR as indicated above for the generation of the $\mathrm{CrzA}^{\triangle \mathrm{CDD} 2}$-GFP coding mutant allele, using as template plasmid pGEXCrzA.

CrzA or PalA glutathione S-transferase fusions were expressed using standard protocols using IPTG as inducer mainly following manufacturer instructions. Pull-down experiments were done essentially as described in (Vincent et al., 2003).

Deletion of GSK3 $\beta$ homologue in A. nidulans

The gene encoding for the putative homologue of glycerol synthase kinase $3 \beta$, named here gskA, was identified in the A. nidulans genome database, http://www.aspgd.org/. BlastP and tBlastN searches using human GSK3 $\beta$ (P49841) and Saccharomyces cerevisiae RIM11 (DAA10036) identified AN6508 as the GSK3 $\beta$ homologue, showing 70\% and 59.7\% amino acid sequence conservation, respectively. Deletion of gskA CDS was completed following our standardized protocol using $r i b o B^{A f}$ as the selectable marker (Markina-Inarrairaegui et al., 2011). Briefly, approximately $1.5 \mathrm{~kb}$ of 5'- and 3'- UTR of AN6508 were amplified by PCR using genomic DNA and the primer pairs GskA(1)/GskA(2) and GskA(3)/GskA(4), respectively. $r i b o B^{A f}$, was obtained by PCR using primers GskA(2') and GskA(3') from plasmid p1830 (see above). The deletion construct was generated in a three-way PCR by fusion of the upstream and downstream regions of AN6508 together with the ribo $B^{A f}$ selection marker. The 
1 homokaryons, several transformants were analyzed by Southern blot and specific radioactive

2 probes (Sambrook et al., 1989) and MAD3844 was selected for this study.

3

$4 \quad$ Protein extraction and analysis procedures

5 Mycelia, cultured under the different growth conditions indicated, were collected by two 6 different methods: 1) filtered through Miracloth (Calbiochem) (Miracloth/filtration procedure,

7 MF), squeezed to dry and frozen in dry ice or 2) vacuum filtered (vacuum/filtration procedure,

8 VF) using $0.45 \mu \mathrm{m}$ pore size nitrocellulose membranes (Scharlau) and frozen in dry ice. In both

9 cases, frozen samples were lyophilized for 16 hours before protein extraction.

10 Two different protocols were followed for obtaining total protein extracts from lyophilized

11 samples. To prevent protein degradation, the alkaline lysis (AL) extraction procedure was used

12 as described in (Hervás-Aguilar and Peñalva, 2010). Essentially, $6 \mathrm{mg}$ of pulverized mycelium

13 was resuspended in $1 \mathrm{ml}$ buffer $(0.2 \mathrm{M} \mathrm{NaOH}, 0.2 \% \beta$-mercaptoethanol) and usually $5 \mu \mathrm{L}$ of

14 each sample were loaded on polyacrylamide gels for protein-content evaluation and estimation

15 of concentration. For those samples requiring further analyses, proteins were extracted as

16 described by Fernández-Martínez et al. (2003) using A50 buffer (25 mM Hepes pH 7.5, 0.1 M

17 EDTA, $50 \mathrm{mM} \mathrm{KCl}, 5 \mathrm{mM} \mathrm{MgCl} 2,10 \%$ glycerol, $0.5 \mathrm{mM}$ DTT) supplemented with $1 \mathrm{mM}$

18 Pefabloc (Roche), $1 \mu \mathrm{M}$ Pepstatin and $0.6 \mu \mathrm{M}$ Leupeptin as proteinase inhibitors. Total protein

19 concentration was determined by Bradford assay (Bradford, 1976) using Bio-Safe ${ }^{\mathrm{TM}}$ Coomasie

20 G-250 Stain from BioRad and following manufacturer's instructions.

21 Combinations of mycelium harvest and protein isolation were as follows: MFA50:

22 Miracloth/filtration procedure followed by protein extraction using A50 buffer, MFAL:

23 Miracloth/filtration procedure followed by alkaline lysis protein extraction, VFA50:

24 Vacuum/filtration procedure followed by protein extraction using A50 buffer and VFAL:

25 Vacuum/filtration procedure followed by alkaline lysis protein extraction.

26 For Western blotting pProteins were resolved in either 10\% SDS-polyacrylamide gels or 4-15\% Mini-PROTEAN ${ }^{\circledR}$ TGX ${ }^{\mathrm{TM}}$ precast polyacrylamide gels (Bio-Rad) and subsequently transferred to nitrocellulose filters using TransBlot ${ }^{\circledR}$ Turbo $^{\mathrm{TM}}$ Transfer System (Bio-Rad). GFP tagged proteins were detected using mouse anti-GFP (1/5,000; Roche). MYC3-PacC fusion was detected using mouse anti-MYC (1/10,000; Sigma). Actin, detected with mouse anti- $\gamma$ actin antibody (1/50,000; ICN Biomedicals), was used as loading control. Peroxidase conjugated goat anti-mouse IgG immunoglobulin (Jackson ImmunoResearch Laboratories) at 1/4,000 was used as a secondary antibody. Peroxidase activity was detected with Amersham Biosciences ECL kit. 
1 The phosphorylation state of the protein samples was mainly analyzed using the procedure by

2 Fernández-Martínez et al (2003). $400 \mathrm{U}$ of $\lambda$ Protein Phosphatase ( $\lambda$ PP) (NE BioLabs Inc) were

3 added to reaction mixtures containing $100 \mu \mathrm{g}$ of total protein extract in NEBuffer for PMP (50

4 mM HEPES pH 7.5, 0.1 M NaCl, 2 mM DTT and 0.01\% Brij 35) supplemented with $1 \mathrm{mM}$

$5 \mathrm{MnCl}_{2}$. Appropriate controls were set to show that electrophoretic mobility changes were

6 specifically due to phosphatase activity in which no enzyme was added to the reaction or it was

7 inhibited adding $10 \mathrm{mM}$ sodium ortho-vanadate to the reaction mixture. The reactions were

8 incubated for $20 \mathrm{~min}$ at $30^{\circ} \mathrm{C}$ and then stopped with the addition of $10 \%$ trichloroacetic acid and

9 further incubation on ice for 10 minutes. Precipitated proteins were collected by centrifugation

10 and washed twice with ethanol:ether solutions 1:1 and 1:3. Protein pellets were resuspended in

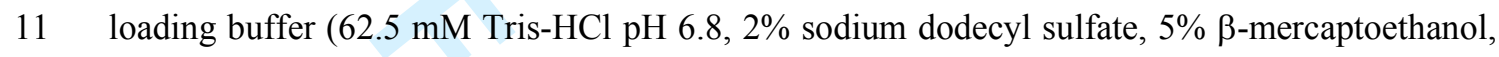

$126 \mathrm{M}$ urea, $0.05 \%$ bromophenol blue) and denatured by heating to $100^{\circ} \mathrm{C}$ for $5 \mathrm{~min}$. For each

13 condition or strain analyzed, $25 \mu \mathrm{g}$ of precipitated proteins were loaded onto polyacrylamide 14 gels.

15 Phosphatase assays using human calcineurin were done following (Heath et al., 2004). For each

16 reaction, $100 \mu \mathrm{g}$ of total protein extract from mycelia of MAD3222 strain were treated with 500

$17 \mathrm{U}$ of recombinant human calcineurin (Calbiochem) together with 2,500 $\mathrm{U}$ of recombinant

18 bovine calmodulin (Sigma) in CP buffer (50 mM Tris-HCl pH 7.5, $1 \mathrm{mM} \mathrm{MgCl}, 1 \mathrm{mM}$ DTT),

19 supplemented with $1 \mathrm{mM}$ Pefablock (Roche), $1 \mu \mathrm{M}$ Pepstatin and $0.6 \mu \mathrm{M}$ Leupeptin as

20 proteinase inhibitors. When indicated $50 \mu \mathrm{M}$ of cyclosporin A was used per assay to inhibit

21 calcineurin activity. Reactions were incubated, stopped and processed for PAGE as indicated

22 above for $\lambda$ PP assays.

Microscopy

25 A. nidulans cells were cultured in appropriately supplemented watch minimal medium (WMM)

26 (Peñalva, 2005) using uncoated $\mu$-Slide 8 well (Ibidi GmbH, Germany) for a direct observation

27 of cultures using a Leica DMI-6000b inverted microscope. For all microscopy experiments,

28 germlings were incubated at $25^{\circ} \mathrm{C}$ for 18 hours except for strain MAD3844 which was 29 incubated at $37^{\circ} \mathrm{C}$ for 20 hours.

30 After this period, the medium was replaced with fresh WMM containing a range of ambient 31 stresses i.e. supplemented with $\mathrm{CaCl}_{2}(50 \mathrm{mM}), \mathrm{MnCl}_{2}(10 \mathrm{mM}), \mathrm{MgCl}_{2}(0.1 \mathrm{M}), \mathrm{NaCl}(0.1 \mathrm{M})$,

$32 \mathrm{KCl}(0.1 \mathrm{M}), \mathrm{NaH}_{2} \mathrm{PO}_{4}(0.1 \mathrm{M})$ or $\mathrm{Na}_{2} \mathrm{HPO}_{4}(0.1 \mathrm{M})$ for adjusting media $\mathrm{pH}$ to 5.2 or 8.0 33 respectively for the last two stimuli.

34 Fluorescence images were acquired using a Leica DMI6000b inverted microscope equipped 35 with a GFP-specific filter ( $\lambda \mathrm{ex}, 470 \mathrm{~nm}$ and $\lambda \mathrm{em}, 525 \mathrm{~nm}$ ), and recorded with an Orca-ER 36 camera (Hamamatsu) driven by the MetaMorph imaging system (Universal Imaging 
1 Corporation). Nuclei were visualized using either following fluorescence of $\mathrm{H} 1-\mathrm{mCh}$ fusion

2 with a TxRed filter or DAPI (4'-6-diamidino-2-phenylindole) staining by replacing the medium

3 with a solution of $60 \mathrm{ng} \mathrm{ml}^{-1}$ of DAPI in fresh WMM also containing $0.01 \%$ Triton to induce

4 entry of the dye in living cells.

5

6

7 Acknowledgements

8 We wish to thank Prof. Gustavo H. Goldman for providing us with null calcineurin mutant 9 strain and to Prof. Claudio Scazzocchio and Dr. Sotiris Amillis for the ckiA mutant collection.

10 We are especially grateful to Elena Reoyo for her assistance and technical advice, and to Dr.

11 Oier Etxebeste for helpful suggestions and critical reading of this manuscript. This work was

12 supported by the Spanish Ministerio de Economia y Competitividad through Grants

13 BFU(2009)-08701 and BFU(2012)-33142 to EAE. P.H-O held a JAE-Pre fellowship from the 14 Spanish Research Council, CSIC.

15

16 
Aramburu,J., Garcia-Cozar,F., Raghavan,A., Okamura,H., Rao,A., and Hogan,P.G. (1998)

\section{References}

Apostolaki,A., Harispe,L., Calcagno-Pizarelli,A.M., Vangelatos,I., Sophianopoulou,V., Arst,H.N. et al. (2012) Aspergillus nidulans CkiA is an essential casein kinase I required for delivery of amino acid transporters to the plasma membrane. Mol Microbiol 84: 530-549.

Selective inhibition of NFAT activation by a peptide spanning the calcineurin targeting site of NFAT. Mol Cell 1: 627-637.

Aramburu,J., Rao,A., and Klee,C.B. (2000) Calcineurin: from structure to function. Curr Top Cell Regul 36: 237-295.

Beals,C.R., Sheridan,C.M., Turck,C.W., Gardner,P., and Crabtree,G.R. (1997) Nuclear export of NF-ATc enhanced by glycogen synthase kinase-3. Science 275: 1930-1934.

Boustany,L.M., and Cyert,M.S. (2002) Calcineurin-dependent regulation of Crzlp nuclear export requires Msn5p and a conserved calcineurin docking site. Genes Dev 16: 608-619.

Chang,P.K. (2008) Aspergillus parasiticus crzA, which encodes calcineurin response zinc-finger protein, is required for aflatoxin production under calcium stress. Int J Mol Sci 9: 2027-2043.

Chin,D., and Means,A.R. (2000) Calmodulin: a prototypical calcium sensor. Trends Cell Biol 10: $322-328$.

Clapham,D.E. (2007) Calcium signaling. Cell 131: 1047-1058.

Cove,D.J. (1966) The induction and repression of nitrate reductase in the fungus Aspergillus nidulans. Biochim Biophys Acta 113: 51-56.

Crabtree,G.R., and Olson,E.N. (2002) NFAT signaling: choreographing the social lives of cells. Cell 109 Suppl: S67-S79.

Cramer,R.A., Jr., Perfect,B.Z., Pinchai,N., Park,S., Perlin,D.S., Asfaw,Y.G. et al. (2008) Calcineurin target CrzA regulates conidial germination, hyphal growth, and pathogenesis of Aspergillus fumigatus. Eukaryot Cell 7: 1085-1097.

Cyert,M.S. (2003) Calcineurin signaling in Saccharomyces cerevisiae: how yeast go crazy in response to stress. Biochem Biophys Res Commun 311: 1143-1150.

De Souza,C.P., Hashmi,S.B., Osmani,A.H., Andrews,P., Ringelberg,C.S., Dunlap,J.C., and Osmani,S.A. (2013) Functional analysis of the Aspergillus nidulans kinome. PLoS One 8: e58008.

Etxebeste,O., Markina-Inarrairaegui,A., Garzia,A., Herrero-Garcia,E., Ugalde,U., and Espeso,E.A. (2009) KapI, a non-essential member of the Pselp/Imp5 karyopherin family, controls colonial and asexual development in Aspergillus nidulans. Microbiology 155: 39343945.

Fernández-Martínez,J., Brown,C.V., Diez,E., Tilburn,J., Arst,H.N., Peñalva,M.A., and Espeso,E.A. (2003) Overlap of nuclear localisation signal and specific DNA-binding residues within the zinc finger domain of PacC. J Mol Biol 334: 667-684. 
1 Findon,H., Calcagno-Pizarelli,A.M., Martinez,J.L., Spielvogel,A., Markina-Inarrairaegui,A., 2 Indrakumar,T. et al. (2010) Analysis of a novel calcium auxotrophy in Aspergillus nidulans. 3 Fungal Genet Biol 47: 647-655.

4 Fiol,C.J., Mahrenholz,A.M., Wang,Y., Roeske,R.W., and Roach,P.J. (1987) Formation of 5 protein kinase recognition sites by covalent modification of the substrate. Molecular mechanism 6 for the synergistic action of casein kinase II and glycogen synthase kinase 3. J Biol Chem 262: $7 \quad 14042-14048$.

8 Fox,D.S., and Heitman,J. (2002) Good fungi gone bad: the corruption of calcineurin. Bioessays 9 24: 894-903.

10 Guerini,D. (1997) Calcineurin: not just a simple protein phosphatase. Biochem Biophys Res 11 Commun 235: 271-275.

12 Gwack,Y., Sharma,S., Nardone,J., Tanasa,B., Iuga,A., Srikanth,S. et al. (2006) A genome-wide 13 Drosophila RNAi screen identifies DYRK-family kinases as regulators of NFAT. Nature 441: 14 646-650.

15 Hagiwara,D., Kondo,A., Fujioka,T., and Abe,K. (2008) Functional analysis of C2H2 zinc finger 16 transcription factor CrzA involved in calcium signaling in Aspergillus nidulans. Curr Genet 54: $17 \quad 325-338$.

18 Heath,V.L., Shaw,S.L., Roy,S., and Cyert,M.S. (2004) Hph1p and Hph2p, novel components of

Hervás-Aguilar,A., and Peñalva,M.A. (2010) Endocytic machinery protein SlaB is dispensable for polarity establishment but necessary for polarity maintenance in hyphal tip cells of Aspergillus nidulans. Eukaryot Cell 9: 1504-1518.

Holmberg,C.I., Tran,S.E., Eriksson,J.E., and Sistonen,L. (2002) Multisite phosphorylation provides sophisticated regulation of transcription factors. Trends Biochem Sci 27: 619-627.

Kafadar,K.A., and Cyert,M.S. (2004) Integration of stress responses: modulation of calcineurin signaling in Saccharomyces cerevisiae by protein kinase A. Eukaryot Cell 3: 1147-1153.

Kafadar,K.A., Zhu,H., Snyder,M., and Cyert,M.S. (2003) Negative regulation of calcineurin signaling by Hrr25p, a yeast homolog of casein kinase I. Genes Dev 17: 2698-2708.

Karababa,M., Valentino,E., Pardini,G., Coste,A.T., Bille,J., and Sanglard,D. (2006) CRZ1, a target of the calcineurin pathway in Candida albicans. Mol Microbiol 59: 1429-1451.

Kullas,A.L., Martin,S.J., and Davis,D. (2007) Adaptation to environmental pH: integrating the Rim101 and calcineurin signal transduction pathways. Mol Microbiol 66: 858-871.

Lev,S., Desmarini,D., Chayakulkeeree,M., Sorrell,T.C., and Djordjevic,J.T. (2012) The $\mathrm{Crz1/Sp1}$ transcription factor of Cryptococcus neoformans is activated by Calcineurin and regulates cell wall integrity. PLoS One 7: e51403.

Li,H., Rao,A., and Hogan,P.G. (2011) Interaction of calcineurin with substrates and targeting proteins. Trends Cell Biol 21: 91-103.

Markina-Inarrairaegui,A., Etxebeste,O., Herrero-Garcia,E., Araújo-Bazán,L., FernándezMartínez,J., Flores,J.A. et al. (2011) Nuclear transporters in a multinucleated organism: functional and localization analyses in Aspergillus nidulans. Mol Biol Cell 22: 3874-3886. 
1 Matheos,D.P., Kingsbury,T.J., Ahsan,U.S., and Cunningham,K.W. (1997) Tcn1p/Crz1p, a 2 calcineurin-dependent transcription factor that differentially regulates gene expression in 3 Saccharomyces cerevisiae. Genes Dev 11: 3445-3458.

4 Mingot,J.M., Espeso,E.A., Diez,E., and Peñalva,M.A. (2001) Ambient pH signaling regulates 5 nuclear localization of the Aspergillus nidulans PacC transcription factor. Mol Cell Biol 21: 6 1688-1699.

7 Mitchell,A.P., and Bowdish,K.S. (1992) Selection for early meiotic mutants in yeast. Genetics 8 131: $65-72$.

9 Nayak,T., Szewczyk,E., Oakley,C.E., Osmani,A., Ukil,L., Murray,S.L. et al. (2006) A versatile 10 and efficient gene-targeting system for Aspergillus nidulans. Genetics 172: 1557-1566.

11 Okamura,H., Aramburu,J., Garcia-Rodriguez,C., Viola,J.P., Raghavan,A., Tahiliani,M. et al. 12 (2000) Concerted dephosphorylation of the transcription factor NFAT1 induces a 13 conformational switch that regulates transcriptional activity. Mol Cell 6: 539-550.

14 Orejas,M., Espeso,E.A., Tilburn,J., Sarkar,S., Arst,H.N., and Peñalva,M.A. (1995) Activation of 15 the Aspergillus $\mathrm{PacC}$ transcription factor in response to alkaline ambient $\mathrm{pH}$ requires proteolysis 16 of the carboxy-terminal moiety. Genes Dev 9: 1622-1632.

17 Pantazopoulou,A., and Peñalva,M.A. (2009) Organization and dynamics of the Aspergillus 18 nidulans Golgi during apical extension and mitosis. Mol Biol Cell 20: 4335-4347.

Polizotto,R.S., and Cyert,M.S. (2001) Calcineurin-dependent nuclear import of the transcription factor Crz1p requires Nmd5p. J Cell Biol 154: 951-960. Comparative genomics of MAP kinase and calcium-calcineurin signalling components in plant and human pathogenic fungi. Fungal Genet Biol 46: 287-298.

Rodriguez,A., Roy,J., Martinez-Martinez,S., Lopez-Maderuelo,M.D., Nino-Moreno,P., Orti,L. et al. (2009) A conserved docking surface on calcineurin mediates interaction with substrates and immunosuppressants. Mol Cell 33: 616-626.

Rodriguez-Galan,O., Galindo,A., Hervás-Aguilar,A., Arst,H.N., and Peñalva,M.A. (2009) Physiological involvement in $\mathrm{pH}$ signaling of Vps24-mediated recruitment of Aspergillus PalB cysteine protease to ESCRT-III. J Biol Chem 284: 4404-4412.

Roy,J., Li,H., Hogan,P.G., and Cyert,M.S. (2007) A conserved docking site modulates substrate affinity for calcineurin, signaling output, and in vivo function. Mol Cell 25: 889-901.

Rubin-Bejerano,I., Sagee,S., Friedman,O., Pnueli,L., and Kassir,Y. (2004) The in vivo activity of Ime1, the key transcriptional activator of meiosis-specific genes in Saccharomyces cerevisiae, is inhibited by the cyclic AMP/protein kinase A signal pathway through the glycogen synthase kinase 3-beta homolog Rim11. Mol Cell Biol 24: 6967-6979. 
1 Soriani,F.M., Malavazi,I., Silva Ferreira,M.E., Savoldi,M., Zeska Kress,M.R., Souza 2 Goldman,M.H. et al. (2008) Functional characterization of the Aspergillus fumigatus CRZ1 3 homologue, CrzA. Mol Microbiol 67: 1274-1291.

4 Spielvogel,A., Findon,H., Arst,H.N., Araújo-Bazán,L., Hernández-Ortiz,P., Stahl,U. et al. 5 (2008) Two zinc finger transcription factors, CrzA and SltA, are involved in cation 6 homoeostasis and detoxification in Aspergillus nidulans. Biochem J 414: 419-429.

7 Stathopoulos-Gerontides,A., Guo,J.J., and Cyert,M.S. (1999) Yeast calcineurin regulates 8 nuclear localization of the Crzlp transcription factor through dephosphorylation. Genes Dev 13: 9 798-803.

10 Stie,J., and Fox,D.S. (2008) Calcineurin regulation in fungi and beyond. Eukaryot Cell 7: 17711186.

12 Thompson,J.D., Gibson,T.J., Plewniak,F., Jeanmougin,F., and Higgins,D.G. (1997) The 13 CLUSTAL_X windows interface: flexible strategies for multiple sequence alignment aided by 14 quality analysis tools. Nucleic Acids Res 25: 4876-4882.

15 Tilburn,J., Scazzocchio,C., Taylor,G.G., Zabicky-Zissman,J.H., Lockington,R.A., and 16 Davies,R.W. (1983) Transformation by integration in Aspergillus nidulans. Gene 26: 205-221.

17 Vincent,O., Rainbow,L., Tilburn,J., Arst,H.N., and Peñalva,M.A. (2003) YPXL/I is a protein 18 interaction motif recognized by Aspergillus PalA and its human homologue, AIP1/Alix. Mol 19 Cell Biol 23: 1647-1655.

20 Wang,H., Liang,Y., Zhang,B., Zheng,W., Xing,L., and Li,M. (2011) Alkaline stress triggers an 21 immediate calcium fluctuation in Candida albicans mediated by Rim101p and Crzlp 22 transcription factors. FEMS Yeast Res 11: 430-439.

23 Zhu,J., Shibasaki,F., Price,R., Guillemot,J.C., Yano,T., Dotsch,V. et al. (1998) Intramolecular 
1 Table 1. List of strains used in this work

\begin{tabular}{|c|c|c|}
\hline Strain & Relevant genotype & Source \\
\hline MAD1425 & pyrG89 & $\begin{array}{l}\text { (Markina- } \\
\text { Inarrairaegui et } \\
\text { al., 2011) }\end{array}$ \\
\hline MAD1739 & pyrG89, pyroA4 & $\begin{array}{l}\text { (Findon et al., } \\
\text { 2010) }\end{array}$ \\
\hline CNA1 & $\triangle c n a A:: p y r o A^{A f}$ & $\begin{array}{l}\text { (Soriani et al., } \\
\text { 2008) }\end{array}$ \\
\hline VIE050 & thiA $A^{p}:$ ckiA & $\begin{array}{l}\text { (Apostolaki et al., } \\
\text { 2012) }\end{array}$ \\
\hline MAD1775 & $\triangle$ palB::pyroA $A^{A f} ;$ pacC 900 & $\begin{array}{l}\text { (Rodriguez-Galan } \\
\text { et al., 2009) }\end{array}$ \\
\hline MAD2446 & hhoA::mCh::pyro $A^{A f}$, pyrG89 & $\begin{array}{l}\text { (Etxebeste } \text { et al., } \\
\text { 2009) }\end{array}$ \\
\hline MAD2449 & $\triangle c r z A:: p y r 4^{N c}$, pyroA4 & This work \\
\hline MAD3709 & $\triangle c r z A:: r i b o B^{A f}$ & This work \\
\hline MAD3020 & $\operatorname{crzA}: \because g f p:: p y r G^{A f}$, riboB2 & This work \\
\hline MAD3021 & $c r z A: \because g f p:: p y r G^{A f}$ & This work \\
\hline MAD4366 & cnaA $:: g f p:: p y r G^{A f}$ & This work \\
\hline MAD3222 & $\triangle c n a A: \because p y r o A ;$ crzA $: \because g f p:: p y r G^{A f}$ & This work \\
\hline MAD4641 & hhoA::mCh::pyroA$A^{A f} ;$ crzA $:: g f p:: p y r G^{A f}$ & This work \\
\hline MAD2166 & pyroA4 [pyroA*::gfp::crzA(M1-K730)] & This work \\
\hline MAD3493 & pyroA4 $\left[\right.$ pyro $\left.^{*}:: g f p:: c r z A(\mathrm{M} 1-\mathrm{L} 612)\right]$ & This work \\
\hline MAD2168 & pyroA4 [pyroA*::gfp::crzA(M1-R448)] & This work \\
\hline MAD2169 & pyroA4 [pyroA* $:: g f p:: c r z A(\mathrm{MD}+\mathrm{P} 445-\mathrm{K} 730)]$ & This work \\
\hline MAD3648 & pyroA4 [pyroA*::gfp::crzA(MD+P445-L612)] & This work \\
\hline MAD2170 & pyroA4 [pyroA*::gfp::crzA(ME+Q608-K730)] & This work \\
\hline MAD2171 & pyroA4 $[$ pyroA $*:: g f p:: c r z A(\mathrm{M} 1-\mathrm{K} 730)] ; \Delta c r z A:: p y r 4^{N_{c}}$ & This work \\
\hline MAD3494 & pyroA4 [pyroA*:::gfp::crzA(M1-L612)]; $\Delta c r z A:: p y r 4^{N c}$ & This work \\
\hline MAD2172 & pyroA4 [pyroA*::gfp::crzA(M1-R448)]; $\Delta c r z A: \because p y r 4^{N c}$ & This work \\
\hline MAD3386 & pyroA4 [pyroA*::gfp::crzA(MD+P445-K730)]; $\Delta c r z A:: p y r 4^{N c}$ & This work \\
\hline MAD3649 & pyroA4 [pyroA*::gfp::crzA(MD+P445-L612)]; $\Delta c r z A: \because p y r 4^{N c}$ & This work \\
\hline MAD3388 & pyroA4 [pyroA* *:gfp::crzA(ME+Q608-K730)]; $\Delta c r z A:: p y r 4^{N c}$ & This work \\
\hline MAD3842 & 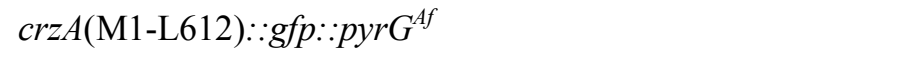 & This work \\
\hline MAD3843 & $\operatorname{crzA}(\mathrm{MD}+\mathrm{P} 445-\mathrm{K} 730): \because g f p:: p y r G^{A f}$ & This work \\
\hline MAD3806 & $\operatorname{crz} A(\Delta \mathrm{Q} 294-\mathrm{H} 316): \because g f p: \because p y r G^{A f}$ & This work \\
\hline MAD4234 & $c r z A(\Delta \mathrm{M} 356-\mathrm{A} 372): \because g f p:: p y r G^{A f}$ & This work \\
\hline MAD4390 & $c r z A(\Delta \mathrm{Q} 294-\mathrm{H} 316 ; \Delta \mathrm{M} 356-\mathrm{A} 372) \because: g f p:: p y r G^{A f}$ & This work \\
\hline MAD3555 & $\begin{array}{l}\Delta c n a A:: \text { pyro }^{A f} ; \text { pyroA4 }\left[\text { pyro }^{*}:: g f p:: c r z A(\mathrm{M} 1-\mathrm{K} 730)\right] \\
\Delta c r z A:: \text { pyr }^{N c}\end{array}$ & This work \\
\hline MAD3559 & $\begin{array}{l}\Delta c n a A:: p_{p y r o A^{A f}} ; \text { pyroA4 }\left[\text { pyroA }^{*}:: g f p:: c r z A(\mathrm{M} 1-\mathrm{L} 612)\right] \\
\Delta c r z A:: \operatorname{pyr}^{N c}\end{array}$ & This work \\
\hline MAD3527 & $\begin{array}{l}\triangle c n a A:: p y r o A^{A f} ; \text { pyroA4 }\left[\text { pyroA }^{*}:: g f p:: c r z A(\mathrm{MD}+\mathrm{P} 445-\right. \\
\mathrm{K} 730)] ; \Delta c r z A:: \text { pyr }^{\text {Nc }}\end{array}$ & This work \\
\hline
\end{tabular}




\begin{tabular}{|c|c|c|}
\hline MAD2946 & ckiA102; crzA::gfp ::pyrG ${ }^{A f}$ & This work \\
\hline MAD3045 & ckiA1919; crzA::gfp::pyrG $G^{A f}$ & This work \\
\hline MAD3044 & ckiA2; crzA::gfp::pyrG $G^{A f}$ & This work \\
\hline MAD3524 & ckiA2, $\triangle$ cnaA $:$ pyro $A^{A f} ;$ crzA $:: g f p:: p y r G^{A f}$ & This work \\
\hline MAD4675 & thiA $A^{p}:$ ckiA; crzA $\because: g f p: \because p y r G^{A f}$ & This work \\
\hline MAD3844 & $\Delta g s k A: \because r i b o B^{A f} ;$ crzA $:: g f p:: p y r G^{A f}$ & This work \\
\hline
\end{tabular}

1
2 
Figure Legends

Figure 1. Cellular localization of CrzA under different abiotic stress growth conditions. Strains MAD3021 and MAD4641, both expressing a CrzA-GFP, were grown in WMM for $18 \mathrm{~h}$ at $25^{\circ} \mathrm{C}$ and observed by fluorescence microscopy under different stress inducing stimuli. Prior to stress treatments (RC, resting cells), CrzA-GFP is mainly localized in the cytoplasm and excluded of nuclei, as shown by the localization of the nuclear marker histone $\mathrm{H} 1$ tagged with monomeric-cherry fluorescent protein (H1-mCh, strain MAD4641). Medium alkalinization ( $\mathrm{pH}$ 8) promoted the accumulation of fluorescence in nuclei while acidification ( $\mathrm{pH} 5.2)$ did not induce changes in the location of CrzA. Supplementation with either $\mathrm{Ca}^{2+}$ or $\mathrm{Mn}^{2+}$ induced nuclear accumulation of $\mathrm{CrzA}$, while after the addition of $\mathrm{Mg}^{2+} \mathrm{CrzA}$ remained cytoplasmic. Elevated concentrations of $\mathrm{Na}^{+}$and $\mathrm{K}^{+}$in the medium caused a distortion of green fluorescence in the cytoplasm but did not stimulate the translocation of CrzA to the nucleus. Nuclei of cells from strain MAD3021 were visualized by DAPI staining after addition of $\mathrm{Na}^{+}$or $\mathrm{K}^{+}$. Scale bar, $5 \mu \mathrm{m}$.

Figure 2. Phenotypic analysis of truncated versions of CrzA. (A) On the left, a diagram showing a comparison of the protein structure of $S$. cerevisiae Crzlp and A. nidulans CrzA followed by a schematic representation of the CrzA truncated forms generated in this work. Initial and final amino acids for each construct are indicated. DBD, orange box, indicates the DNA binding domain. The green box labels the GFP tag. On the right, growth tests of mutant strains expressing the different CrzA truncated forms under the control of the gpdA promoter either in a wild-type or null $c r z A$ genetic background. Strains were inoculated on standard MMA (-), MMA supplemented with calcium $\left(\mathrm{Ca}^{2+}\right)$ or buffered to alkaline $\mathrm{pH}(\mathrm{pH} 8)$. Images of colonies were taken after 2 days of incubation at $37^{\circ} \mathrm{C}$. Strains used in this study were MAD2166, MAD3493, MAD2169, MAD2168, MAD3648, MAD2170, MAD1425, MAD2171, MAD3494, MAD3386, MAD3386, MAD2172, MAD3649, MAD3388, MAD2449. (B) On the left, a schematic representation of the construction of the GFP-tagged (green box) truncated forms expressed under the $c r z A$ native promoter (square-filled box). On the right, growth tests of strains using MMA (-), alkaline medium ( $\mathrm{pH}$ 8) or supplemented with increasing molar concentrations of $\mathrm{Ca}^{2+}$. Images of colonies were taken after 2 days of incubation at $37^{\circ} \mathrm{C}$ Strains used in these experiments were: MAD1425, MAD3709, MAD3021, MAD3842, MAD3843. (C) Comparison of the cellular distribution of the GFP-tagged CrzA mutant proteins under stressing and non stressing conditions. Conidiospores were cultured in $\mathrm{WMM}$ for $18 \mathrm{~h}$ at $25^{\circ} \mathrm{C}$ and photographed at basal conditions, RC (resting cells), after a shift to fresh WMM supplemented with $\mathrm{Ca}^{2+}\left(50 \mathrm{mM} \mathrm{CaCl}_{2}\right)$ or WMM buffered at alkaline $\mathrm{pH}$ (pH 8). Strains used, from left to right: MAD2166, MAD3493, MAD2169, MAD2168, MAD3648, MAD2170. Scale bar, 5 mm. 
2 Figure 3. Calcineurin-dependent signaling of CrzA. (A) Growth tests of GFP-tagged and untagged-CrzA strains under calcium or alkaline $\mathrm{pH}$ stress. Media and culture conditions are as in Fig. 2. Strains used here were MAD1425, CNA1, MAD3021, MAD3222 and MAD3709. (B) Calcineurin is required for the nuclear accumulation of CrzA under stressing growth conditions. Microscopy observations were conducted as in Fig. 2C. Strains used: MAD3021 and MAD3222. Scale bar, $5 \mu \mathrm{m}$.

Figure 4. Changes in the electrophoretic mobility of CrzA due to calcineurin activity. (A) Western blot showing changes in CrzA-GFP in a null cnaA background or by inhibiting calcineurin using cyclosporine-A (CsA). The concentration of CsA used in each assay is indicated in $\mu \mathrm{M}$ on top of the image. Detection of CrzA in protein extracts of a wild type is shown as control of CrzA-GFP mobility. (B) $\lambda$-Protein phosphatase ( $\lambda$ PP) treatment of protein extracts from a wild-type cnaA strain expressing CrzA-GFP with or without addition of CsA. As control ortho-vanadate (o-vanadate) was used to specifically inhibit $\lambda$ PP reaction. (C) $\lambda$ PP assays showing the change in mobility of CrzA-GFP in a null cnaA background with or without CsA treatment. As control, $10 \mathrm{mM}$ o-vanadate was used to inhibit $\lambda$ PP reaction. (D) Western blot showing the mobility of CrzA-GFP fusion in extracts from a null cnaA strain after phosphatase assays with either recombinant human calcineurin (h $\mathrm{CN}$ ) or $\lambda$ PP. CsA and ovanadate were used to inhibit $\mathrm{h} \mathrm{CN}$ and $\lambda$ PP reactions, respectively (labeled as inhibitor). (E) Electrophoretic mobility of CrzA in protein extracts from a wild-type cnaA strain after addition of calcium $\left(\mathrm{Ca}^{2+}\right)$ or medium alkalinization $(\mathrm{pH} 8)$. Mycelium samples of each condition were taken at the indicated times (min). For all Western blots, detection of CrzA-GFP from wild-type and null cnaA strains were used as control of CrzA mobility. CrzA-GFP fusions were detected using a mouse-monoclonal GFP antibodies mixture. As loading control actin was detected using a commercial polyclonal antibody. Total protein extracts were from mycelia of strains: MAD3021 and MAD3222. Protein extracts for panels A and E were obtained using VFAL protocol (see Supplementary Text and Experimental Procedures section). For panels B, C and D protein extracts were obtained using VFA50 protocol.

Figure 5. Calcineurin regulates nuclear localization and phosphorylated state of CrzA forms of CrzA in the absence and presence of calcineurin function. The wild-type and null cnaA strains expressing the full-length or the CrzA(1-612) form are indistinguishable under basal and stressing conditions. The CrzA(445-730) form remains nuclear at any growth condition or 
1 the CrzA construct under study, including amino acid coordinates. The orange box labels the

2 DBD. RC, resting cells. Scale bar, $5 \mu \mathrm{m}$. (B) $\lambda$ PP assays of functional truncated versions of

3 CrzA. Phosphorylated CrzA(1-612) protein was detected in a null cnaA mutant. No changes in

4 electrophoretic mobility after $\lambda$ PP treatment were detected in the $\mathrm{CrzA}(445-730)$ form. o-

5 vanadate was used to specifically inhibit $\lambda$ PP reaction. GFP-CrzA fusions were detected using

6 a monoclonal antibody against GFP. Protein extracts were obtained using VFA50 protocol. (C)

7 Constitutive expression of full or truncated functional CrzA forms does not complement null

8 cnaA growth defects. Strains were grown for 2 days at $37^{\circ} \mathrm{C}$ on MMA (-) and on MMA

9 supplemented with $\mathrm{Ca}^{2+}$ or buffered at $\mathrm{pH}$ 8. Strains used in $\mathrm{A}, \mathrm{B}$ and $\mathrm{C}$ panels are: MAD2171,

10 MAD3555, MAD3494, MAD3559, MAD3386 and MAD3527.

Figure 6. Functional analyses of identified calcineurin docking domains (CDDs) in CrzA

(A) Diagram of the CDD truncated forms of CrzA studied here. Removal of the violet and light blue rectangles represents the deletion of the CDD1 (sites 1 and 2) and CDD2 (site 3), respectively. DBD, DNA binding domain. On the right it is shown a multiple alignment comprising the three CDD predicted sites in CrzA together with the functional CDD motif present in Crzlp, the alternative low and high affinity CDD sequences also analyzed in Crzlp (Roy et al., 2007), and the consensus sequence of CDDs defined in mammalian NFAT (Aramburu et al., 1998). (B) Multiple sequence alignment of CrzA homologues from filamentous fungi showing the region containing putative CDDs. Dark blue rectangles above the alignment indicate putative CDD sites 1, 2 and 3 identified in A. nidulans CrzA ((Spielvogel et al., 2008), and this work). Violet and light blue rectangles below the alignment illustrate the limits of the region deleted in the mutant CrzA proteins generated. Proteins alignments of the CrzA homologues from Anid: A. nidulans (BAE94327), Aory: Aspergillus oryzae (BAE57003), Afum: Aspergillus fumigatus (EAL88401), Afla: Aspergillus flavus (EED48569), Ncra: Neurospora crassa (EAA32849), Cglo: Chaetomium globosum (EAQ88414) and Gzea: Gibberella zeae (XP_381517) were performed using in-site ClustalX software (Thompson et al., 1997). Shading follows Blosum 32 for similarities in residue composition, white $<60 \%$, light grey $60 \%$, dark grey $80 \%$ and black $100 \%$ conservation. (C) Growth test of mutant $c r z A$ strains lacking the putative CDDs. Strains were inoculated on MMA, alkaline medium or supplemented with increasing molar concentrations of $\mathrm{Ca}^{2+}$ and incubated for 2 days at $37^{\circ} \mathrm{C}$. Deletion of the predicted CDDs did not affect to the survival of the strains. Strains shown from top to bottom: MAD1425, MAD3709, MAD3021, MAD3806, MAD4234, MAD4390 and MAD3222. (D) Localization analysis of the hypothetical CDD defective forms of CrzA. Conidia from strains expressing mutant forms of CrzA were grown in $\mathrm{WMM}$ at $25^{\circ} \mathrm{C}$ for $18 \mathrm{~h}$ and observed by 
1 strain (MAD3806), CrzA localizes to nuclei in response to stimuli. Lack of CDD2 (strain 2 MAD4234) prevents CrzA from nuclear accumulation at any condition. RC, resting cells. Scale 3 bar, $5 \mu \mathrm{m}$. (E) Western analysis showing the changes in mobility of $\Delta$ CDD1+2 CrzA-GFP 4 mutant version. Lanes on the left show the mobility shift due to the deletion of CDD1 and 5 CDD2 sites compared with the wild-type CrzA-GFP fusion, and the effect of CsA treatment 6 reducing the mobility of both wild-type and mutant chimaeras. Lanes on the right show the mobility of $\triangle \mathrm{CDD} 1+2 \mathrm{CrzA}-\mathrm{GFP}$ under stressing conditions. Mobility shifts are observed when the culture was incubated with $\mathrm{CsA}$ or $\mathrm{Ca}^{2+}$ at the incubation times (min) indicated above the panel but no changes were detected with alkalinity. Detection of CrzA-GFP from $\triangle c n a A$ strain MAD3222 was used as control of low mobility of CrzA. Protein extracts were obtained using VFAL protocol. (F) $\lambda$ PP treatment of protein extracts of strain MAD4390 expressing $\Delta \mathrm{CDD} 1+2$ CrzA-GFP. Mycelia were treated with or without CsA prior to protein extraction. As control o-vanadate was used to specifically inhibit $\lambda$ PP reaction The Western-blot image is over-exposed to show the diffuse band of CrzA-GFP fusion detected with the GFP antibody in the absence of CsA treatment. Below it is shown a reduced exposure for detection of $\triangle \mathrm{CDD} 1+2$ CrzA-GFP in protein extracts after CsA treatment. Protein extracts were obtained using VFA50 protocol. (G) Pull-down experiment using bacterially expressed GST-CrzA and GST-CrzA ${ }^{\triangle \mathrm{CDD} 2}$ forms as baits and CnaA-GFP expressed in A. nidulans (strain MAD4366) as prey. The CDD2 motif is necessary for interaction between CnaA and CrzA. I= input of total protein extract from A. nidulans. $\mathrm{B}=$ bound protein fraction. For all Western blots, detection of CrzA-GFP and CnaA-GFP fusions were done using a monoclonal antibody against GFP.

Figure 7. ckiA mutations alters the mobility pattern but not the cellular localization of CrzA. (A) Phenotypic analysis of $c k i A$ mutant strains expressing CrzA-GFP. The $c k i A$ defective strains are not able to grow in MMA containing arginine (Arg, $5 \mathrm{mM})$ as main nitrogen source but are resistant to elevated concentrations of extracellular $\mathrm{Ca}^{2+}$ and alkalinity $(\mathrm{pH} 8$ ). Triple mutant MAD3524 displayed an additive phenotype as a result of the effect of single mutations. (B) Localization of CrzA-GFP in ckiA mutants. Conidiospores from the indicated strains were cultured in WMM at $25^{\circ} \mathrm{C}$ for $18 \mathrm{~h}$ and shifted to fresh medium supplemented with $\mathrm{Ca}^{2+}$ or buffered at $\mathrm{pH}$ 8. None of the strains carrying ckiA mutations exhibit changes in the cellular distribution of CrzA when compared to a ckiA wild-type background at any condition assayed. Scale bar, $5 \mu \mathrm{m}$. (C) Western blot showing differences in the mobility of CrzA in ckiA defective mutants compared with either wild-type or $\triangle c n a A$ genetic backgrounds, and the effect of the calcineurin inhibitor, CsA. Protein extracts were obtained using VFAL protocol. CrzA detection and electrophoresis conditions as those mentioned in legend of Fig. 4. Strains used in 
1 experiments shown in this figure are MAD1425, MAD3709, MAD3021, MAD2946,

2 MAD3045, MAD3044, MAD3524 and MAD3222.

4 Figure 8. CrzA requires the activity of GSK3 $\beta$, GskA, for a proper stimulus response.

5 (A) Schematic representation and distribution of potential Gsk $3 \beta$ phosphorylation sites $(\downarrow)$ along

6 the CrzA amino acid sequence. DBD, orange box, indicates the location of the DNA binding

7 domain. (B) Comparison of colonial growth phenotype of wild-type, null $c r z A$ and null gskA

8 strains. The null gskA strain (MAD3844) shows strong morphological defects on MMA that can

9 be slightly overcome using CMA. The addition of $\mathrm{Ca}^{2+}$ or alkalinization of CMA plates has no

10 major effects on the already poor colonial growth of the null gskA strain. (C) In the absence of

11 GskA, CrzA does not accumulate in nuclei at alkaline pH. Conidiospores of the strain

$12 \mathrm{MAD} 3844$ were cultured in $\mathrm{WMM}$ at $37^{\circ} \mathrm{C}$ for $20 \mathrm{~h}$ and afterwards medium was replaced with

13 fresh WMM supplemented with a stress-inducing agent. The addition of $\mathrm{Ca}^{2+}$ promoted the

14 accumulation of CrzA in the nucleus (each white arrow point to a nucleus before and after

15 calcium stimulation). Alkaline $\mathrm{pH}$ did not enhance the nuclear accumulation of fluorescence.

16 Magnification of a nucleus along media shift shows how the fluorescence was excluded from

17 nucleus in resting cells (RC) and alkalinization induced nuclear entry of fluorescence but not its

18 accumulation. Scale bar, $5 \mu \mathrm{m}$. (D) Western blot analysis showing high mobility bands of the

19 CrzA-GFP chimera from a null gskA strain. A lower mobility band was observed by treatment

20 of the culture with CsA, but it does not reach the mobility of a non-treated wild type extract.

21 Strains used: MAD3844 and MAD3021. (E) $\lambda$ PP assay of CrzA-GFP in a null gskA

22 background. In the absence of CsA no shifts of mobility of CrzA are detected after $\lambda \mathrm{PP}$. When

23 calcineurin activity is inhibited by incubation with CsA in the absence of $g s k A$ expression, CrzA

24 was dephosphorylated in vitro by $\lambda$ PP. O-vanadate was used to inhibit $\lambda$ PP reaction. Mobility

25 of CrzA-GFP from a wild type strains after $\lambda$ PP treatment is shown as control of a low level of

26 phosphorylation of CrzA. Strains used were MAD3844 and MAD3021. (F) Western blot

27 analysis of CrzA-GFP from the gskA mutant under stress inducing conditions. Incubation of the 28 culture (MAD3844) with $\mathrm{Ca}^{2+}$ or alkaline conditions promotes a delay on the mobility of CrzA.

29 For all western blots, CrzA-GFP fusions were detected using a mouse-monoclonal GFP

30 antibodies mixture. For panels D and F protein extracts were obtained using VFAL protocol,

31 and for panel E with VFA50 protocol.

Figure 9. Models for the signaling and nuclear transport regulation of CrzA in $A$. nidulans. 


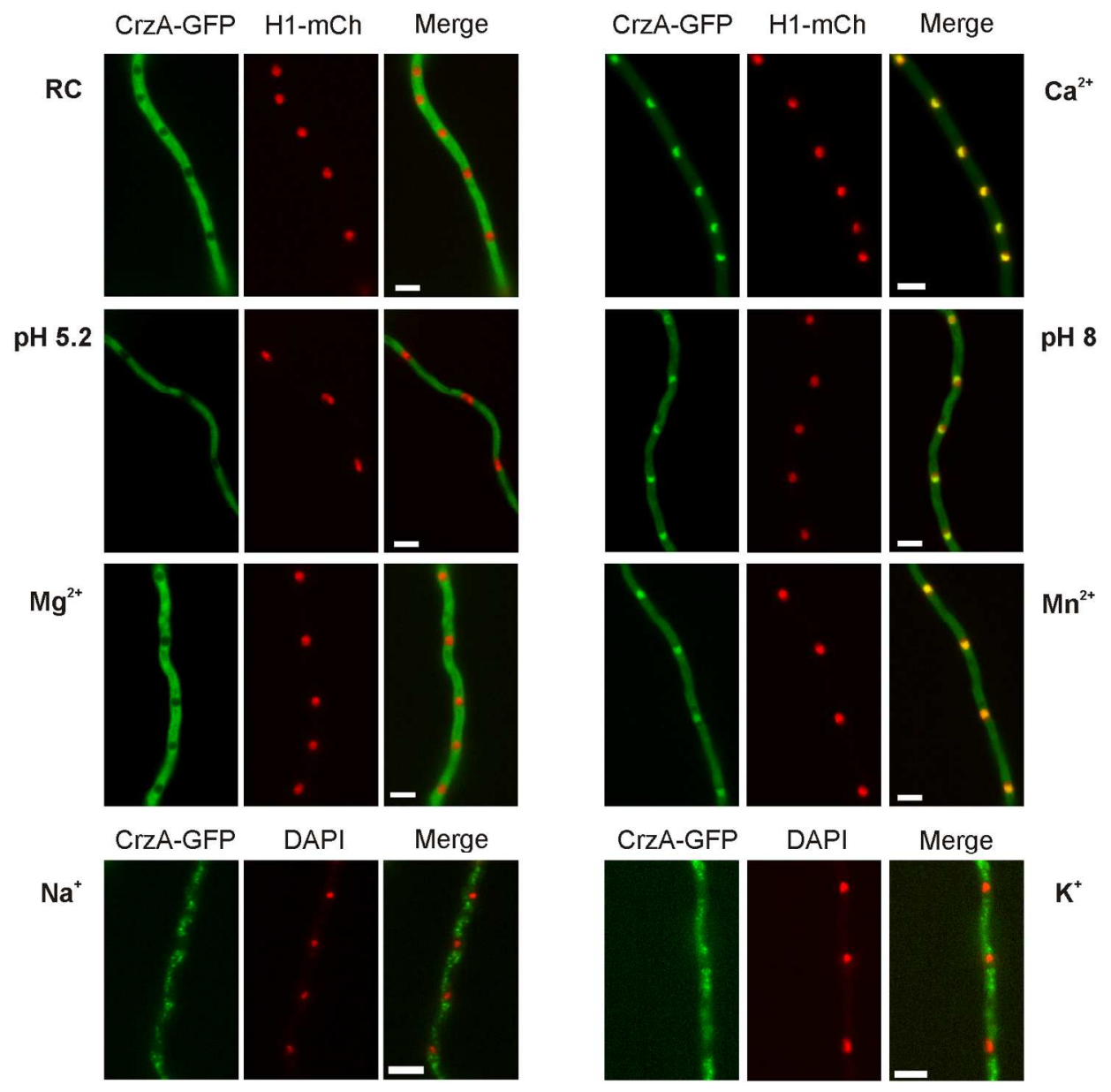

Figure 1. Colour figure

$154 \times 168 \mathrm{~mm}$ (300 x 300 DPI) 


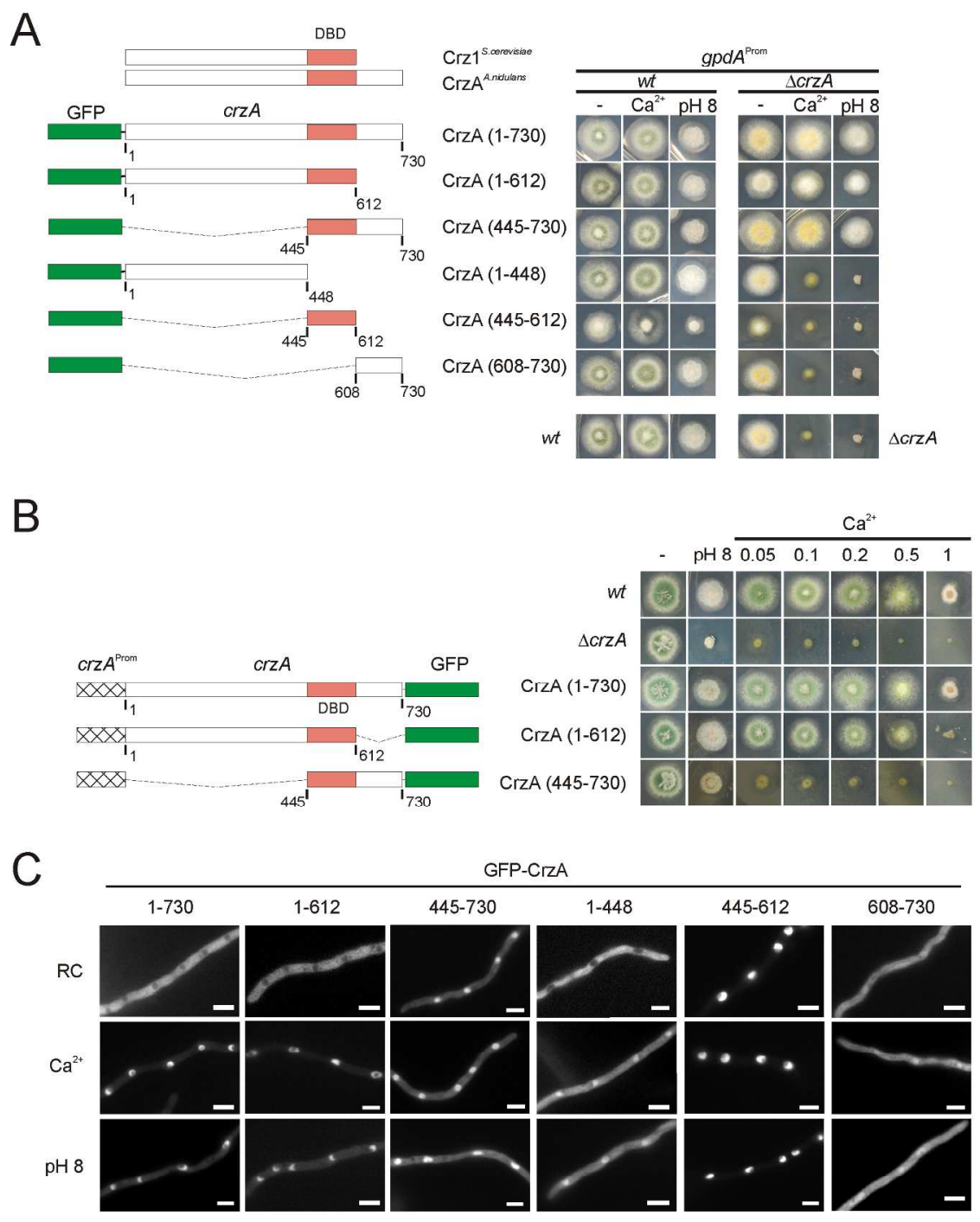

Figure 2, Colour figure $194 \times 253 \mathrm{~mm}$ ( $300 \times 300$ DPI) 

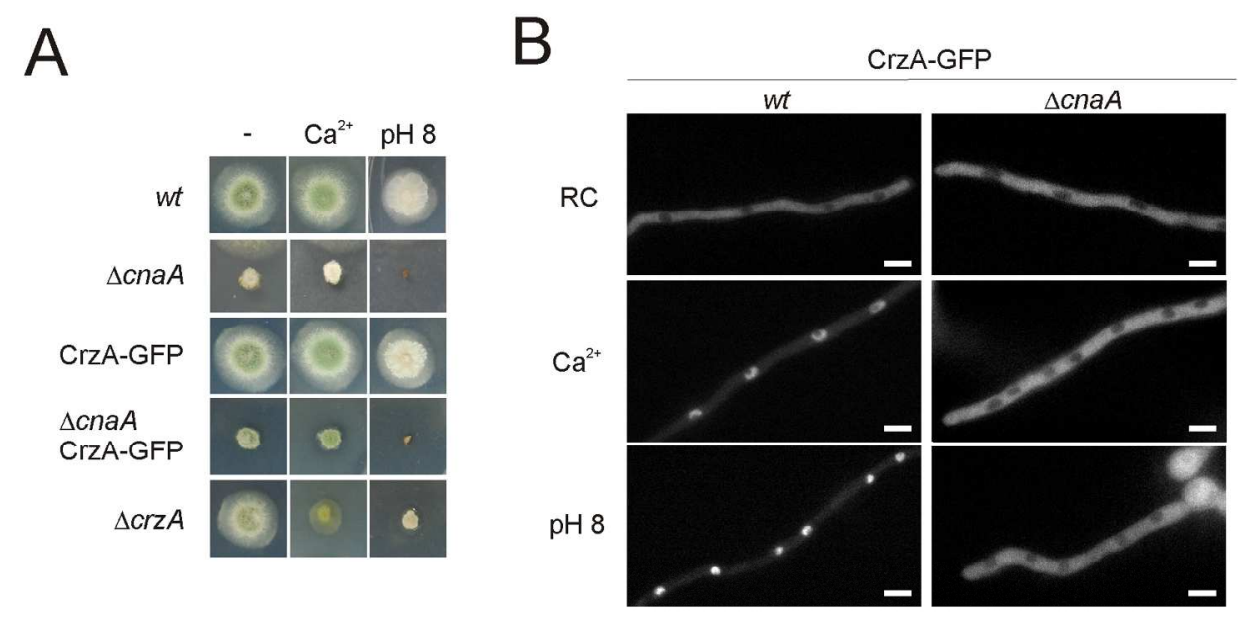

Figure 3. B/W Figure, Colour online $169 \times 91 \mathrm{~mm}(300 \times 300 \mathrm{DPI})$ 
A

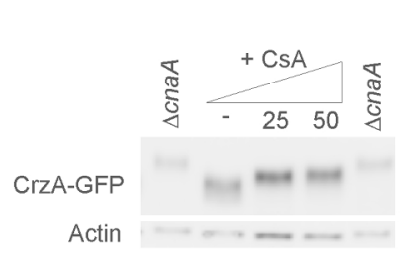

B
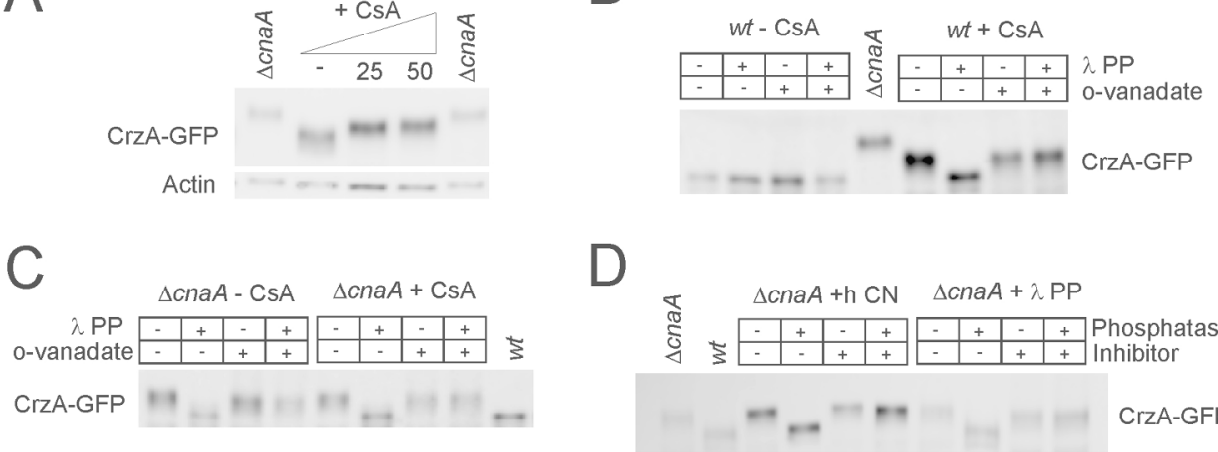

D
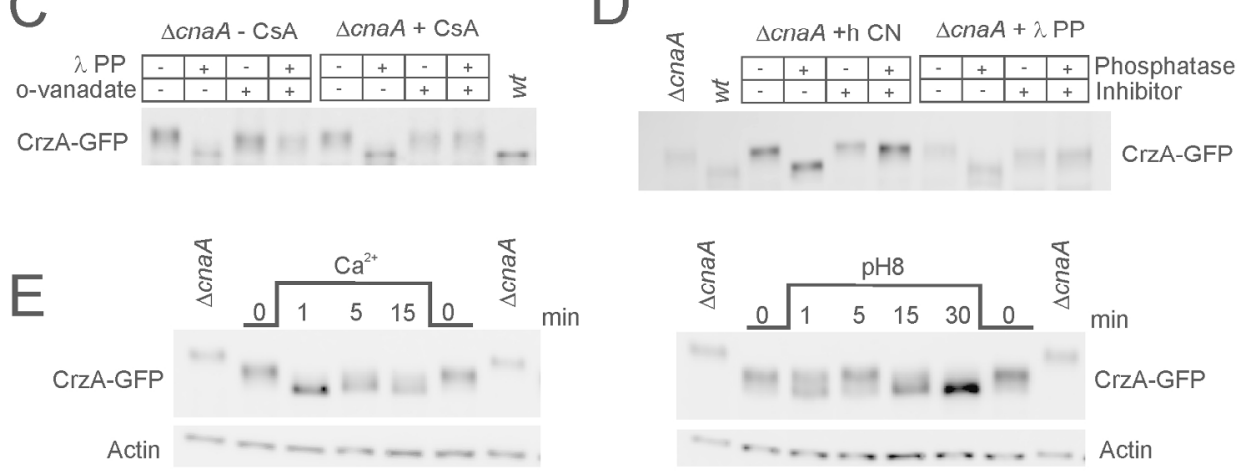

Figure 4. B/W Figure $188 \times 129 \mathrm{~mm}(300 \times 300$ DPI $)$ 


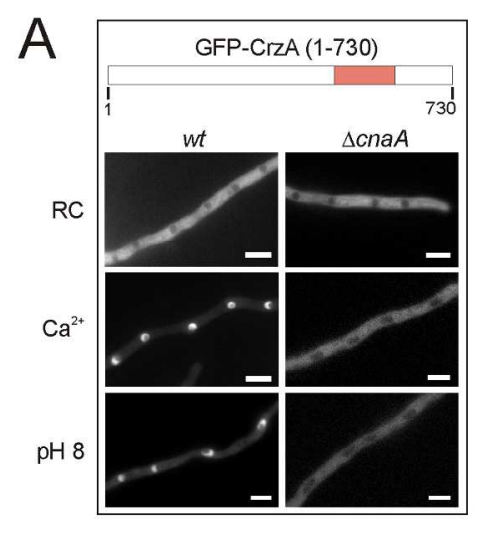

B

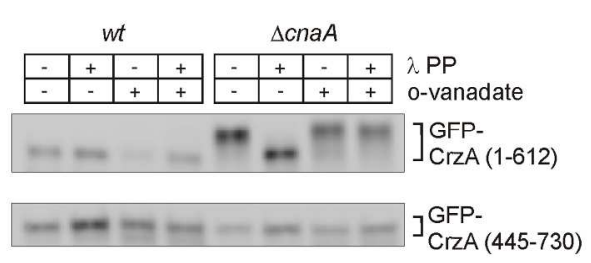

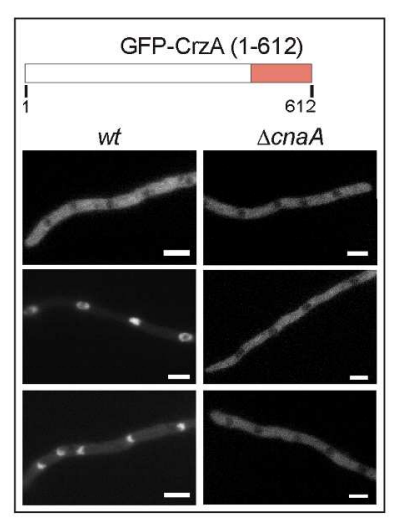

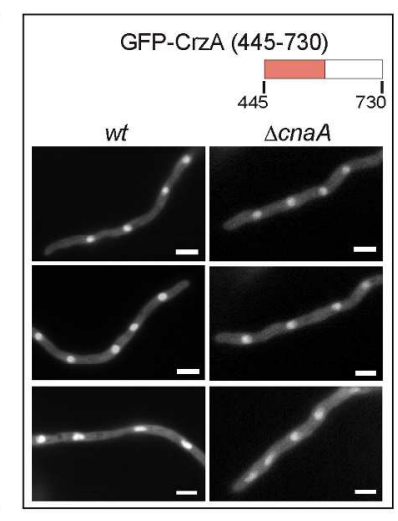

C

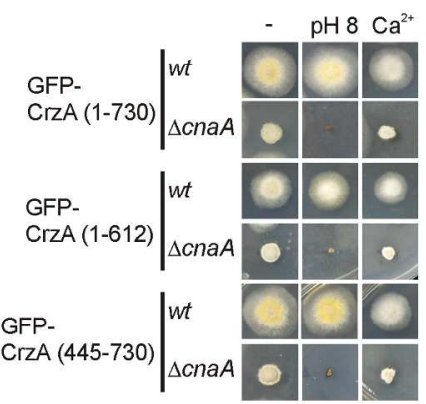

Figure 5. B/W Figure, Colour online $197 \times 156 \mathrm{~mm}$ (300 x 300 DPI) 
A

\begin{tabular}{|c|c|c|c|c|}
\hline & DBD & & site 1 & PYISPQ \\
\hline & & CrzA & $\begin{array}{l}\text { site } 2 \\
\text { site } 3\end{array}$ & $\begin{array}{l}\text { PQIMPQ } \\
\text { PSTNYVE }\end{array}$ \\
\hline I & I & $\mathrm{CrzA}^{\mathrm{ALUU}}$ & crzlp & PIISIQ \\
\hline & ב & $\mathrm{CrZA}^{\mathrm{sCOD2}}$ & $\begin{array}{l}\text { crzlp } \\
\text { crzlp }\end{array}$ & $\begin{array}{l}\text { PVIAVNN } \\
\text { PVIVIT }\end{array}$ \\
\hline & $\square$ & $\mathrm{CrzA}^{\mathrm{sCODOA+2}}$ & NEAT & PxIXIT \\
\hline
\end{tabular}

B

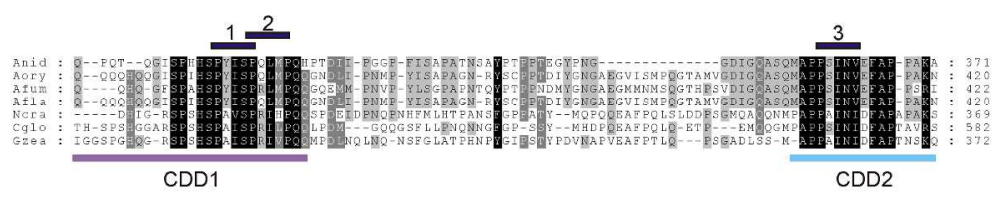

C
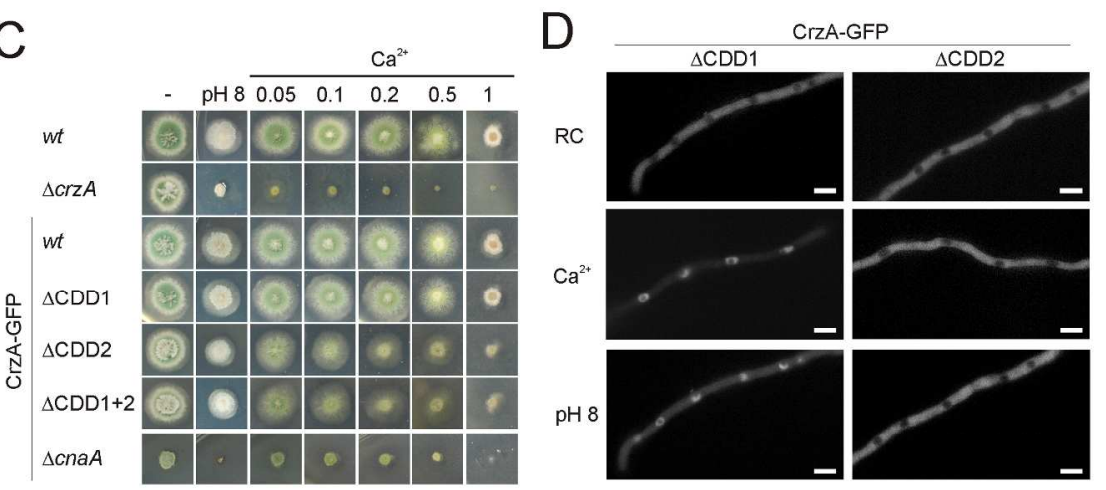

E

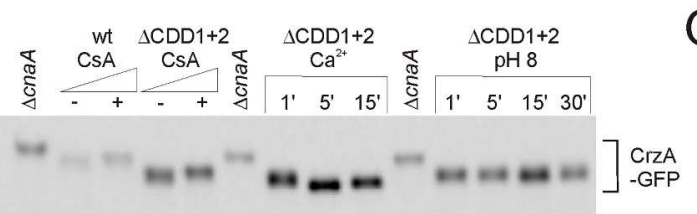

$\mathrm{F}$

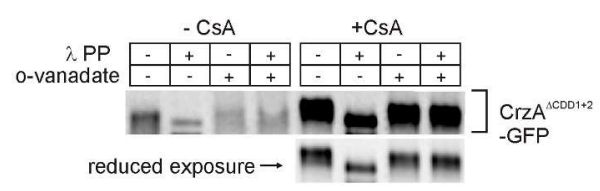

G

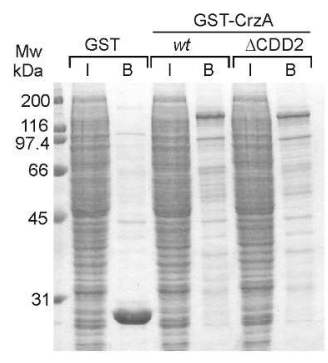

CnaA-GFP - --

Figure 6, B/W Figure, Colour online $196 \times 262 \mathrm{~mm}(300 \times 300 \mathrm{DPI})$ 

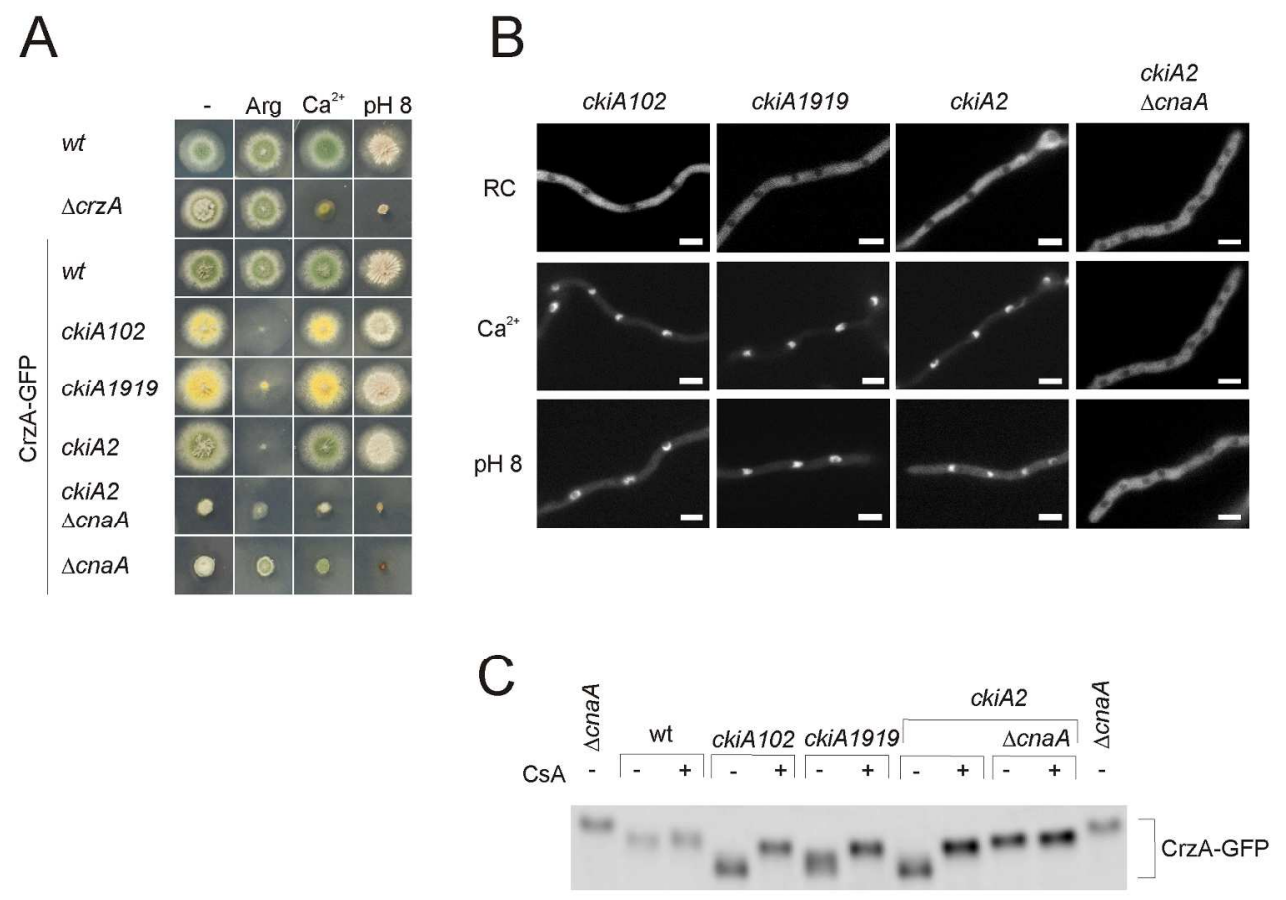

Figure 7. B/W Figure, Colour online $194 \times 142 \mathrm{~mm}(300 \times 300$ DPI $)$ 


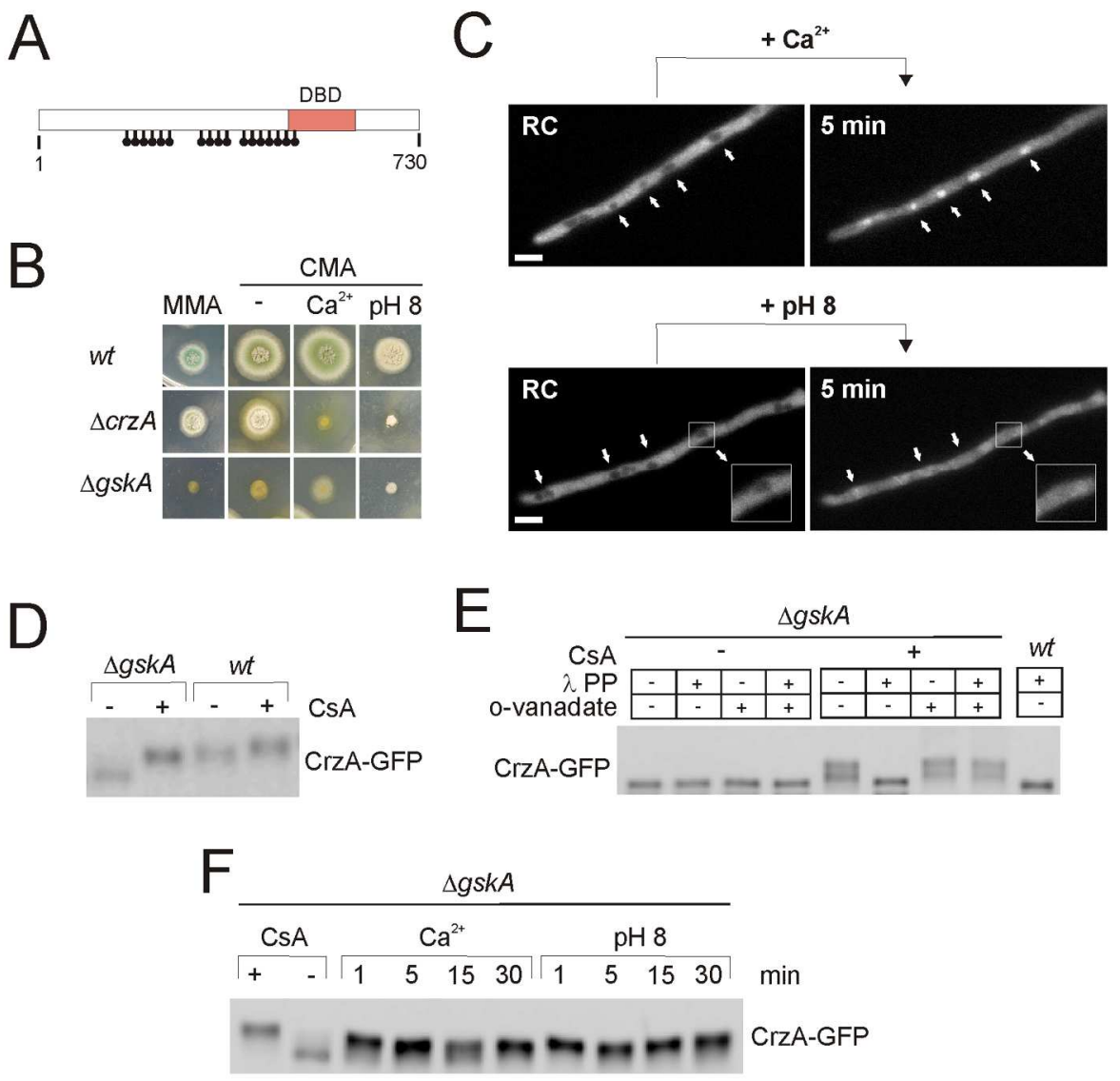

Figure 8 . B/W Figure, Colour online $169 \times 161 \mathrm{~mm}(300 \times 300 \mathrm{DPI})$ 


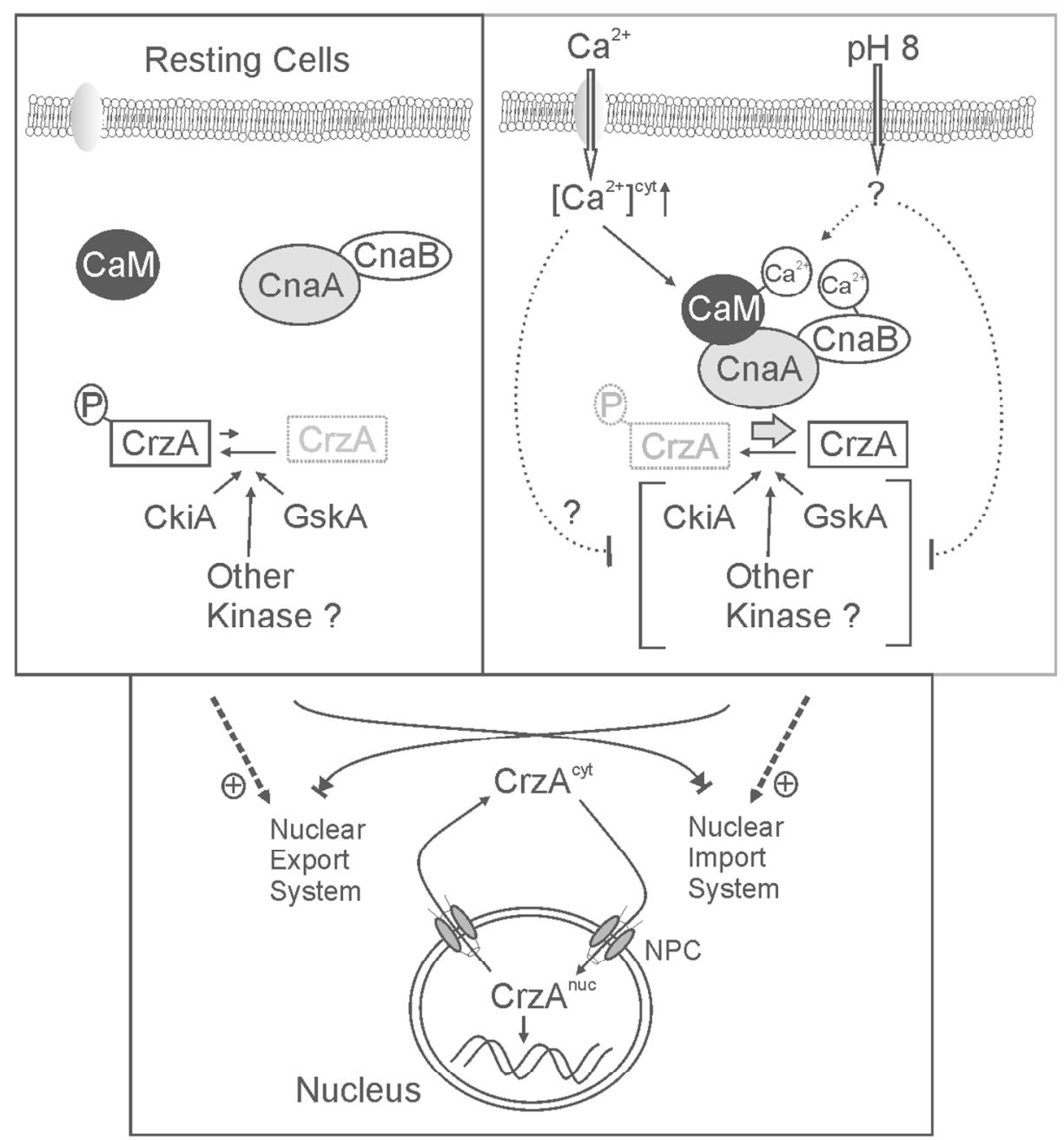

Figure 9. B/W Figure $114 \times 123 \mathrm{~mm}$ (300 x 300 DPI) 